Reviews and perspectives

\title{
Going beyond LTM in the MTL: A synthesis of neuropsychological and neuroimaging findings on the role of the medial temporal lobe in memory and perception
}

\author{
Kim S. Graham ${ }^{\mathrm{a}, *}$, Morgan D. Barense ${ }^{\mathrm{b}}$, Andy C.H. Lee ${ }^{\mathrm{c}}$ \\ a Wales Institute of Cognitive Neuroscience, School of Psychology, Cardiff University, Tower Building, Park Place, Cardiff CF10 $3 A T$, UK \\ ${ }^{\mathrm{b}}$ University of Toronto, Department of Psychology, 100 St. George Street, Toronto, Ontario M5S 3G3, Canada \\ ${ }^{\mathrm{c}}$ Department of Experimental Psychology, University of Oxford, South Parks Road, Oxford OX1 3UD, UK
}

\section{A R T I C L E I N F O}

\section{Article history:}

Received 14 May 2007

Received in revised form 8 December 2009

Accepted 1 January 2010

Available online 13 January 2010

\section{Keywords:}

Memory

Perception

Language

Amnesia

Hippocampus

Perirhinal cortex

Working memory

Neuropsychology

Functional neuroimaging

\begin{abstract}
A B S T R A C T
Studies in rats and non-human primates suggest that medial temporal lobe (MTL) structures play a role in perceptual processing, with the hippocampus necessary for spatial discrimination, and the perirhinal cortex for object discrimination. Until recently, there was little convergent evidence for analogous functional specialisation in humans, or for a role of the MTL in processes beyond long-term memory. A recent series of novel human neuropsychological studies, however, in which paradigms from the animal literature were adapted and extended, have revealed findings remarkably similar to those seen in rats and monkeys. These experiments have demonstrated differential effects of distinct stimulus categories on performance in tasks for which there was no explicit requirement to remember information across trials. There is also accruing complementary evidence from functional neuroimaging that MTL structures show differential patterns of activation for scenes and objects, even on simple visual discrimination tasks. This article reviews some of these key studies and discusses the implications of these new findings for existing accounts of memory. A non-modular view of memory is proposed in which memory and perception depend upon the same anatomically distributed representations (emergent memory account). The limitations and criticisms of this theory are discussed and a number of outstanding questions proposed, including key predictions that can be tested by future studies.
\end{abstract}

(c) 2010 Elsevier Ltd. All rights reserved.

\section{Contents}

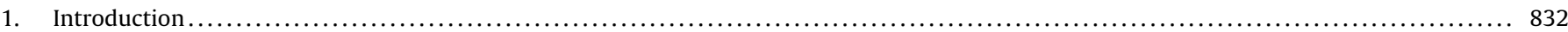

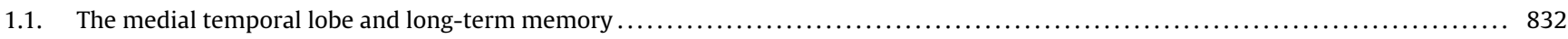

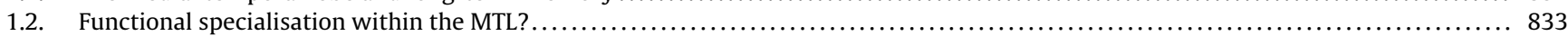

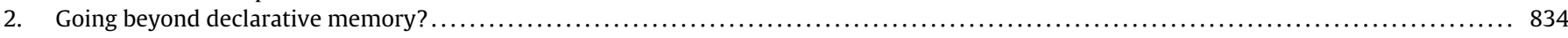

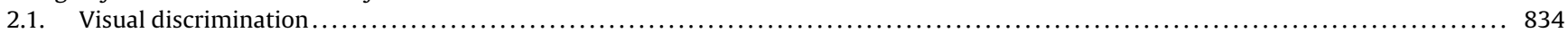

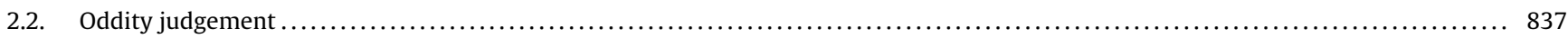

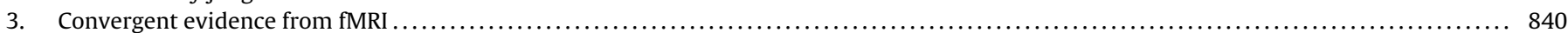

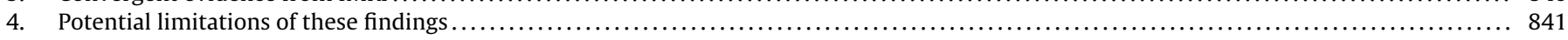

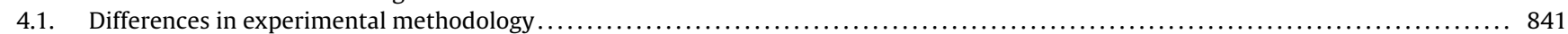

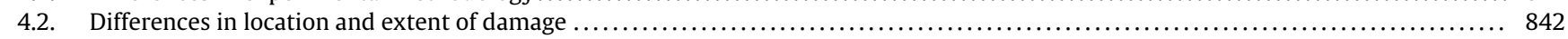

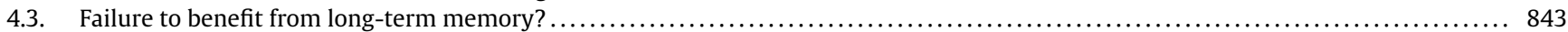

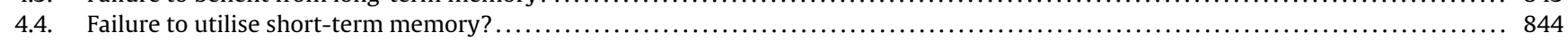

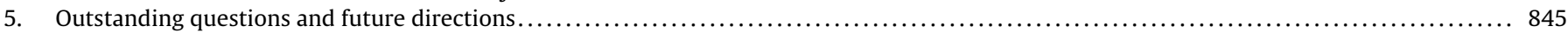

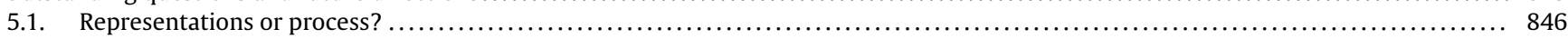

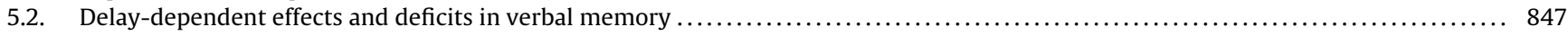

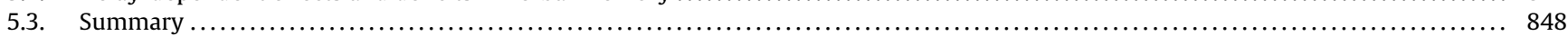

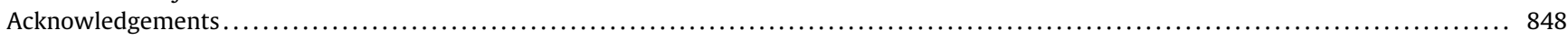

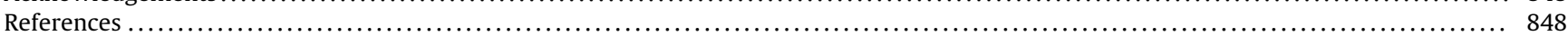

\footnotetext{
* Corresponding author.

E-mail address: grahamks@cardiff.ac.uk (K.S. Graham).
} 


\section{Introduction}

In a recent email exchange, we asked one of our participants with restricted damage to the hippocampus exactly how she thought her memory affected her every-day life. She wrote, "The areas of my life that I find most challenging are when I am given a series of directions, remembering my way around somewhere (familiar or unfamiliar ${ }^{1}$ ), how I got into a building and how I can get out of it again, driving somewhere not only for the first time, but many times, remembering where I left my car and how I got into the car park in the first place, which way to turn out of a car park to get home .... Whichever angle I look, everything looks the same". These comments are consistent with problems in spatial memory and navigation, difficulties that have previously been reported in individuals with damage that includes the hippocampus (Bird, Vargha-Khadem, \& Burgess, 2008; Bohbot, Iaria, \& Petrides, 2004; Bohbot et al., 1998; Burgess, Maguire, \& O’Keefe, 2002; Feigenbaum \& Morris, 2004; Holdstock, Gutnikov, Gaffan, \& Mayes, 2000; King, Burgess, Hartley, Vargha-Khadem, \& O’Keefe, 2002; King, Trinkler, Hartley, Vargha-Khadem, \& Burgess, 2004; Maguire, Nannery, \& Spiers, 2006; Rosenbaum et al., 2000; Rosenbaum, Gao, Richards, Black, \& Moscovitch, 2005; Spiers, Burgess, Hartley, Vargha-Khadem, \& O'Keefe, 2001; Teng \& Squire, 1999). More surprisingly, however, our patient continues her description by saying, "I would prefer not to call my experiences 'memory problems', they are not. This is a total misrepresentation of the damage I have. What I experience are 'orientational problems"'. In addition to describing difficulties with remembering information from moment to moment, therefore, our patient also reports that her memory impairment stems from a more basic problem with determining her position in relation to her surroundings. In describing how she manages this impairment in spatial processing, she writes, "I check my position at regular intervals. I literally take mental photos by stopping, turning round and taking a visual snapshot. When it is time to find my way back, I rely on my mental snapshots. I think my visual memory is good and it compensates for the reduced spatial memory I have. I guess the mental snapshot strategy is a bit like trawling through the photos on a digital camera".

This description highlights a number of features of amnesia that have become of increasing interest to memory scientists over the last few years, and which will be the focus of this review: (a) that the hippocampus may be particularly important for spatial memory and the spatial relationship between objects or oneself within the environment (Bird \& Burgess, 2008a); (b) that injury to particular medial temporal lobe (MTL) structures may differentially affect one type of memory more than another (in the case above, spatial memory seems more impaired than object memory); and (c) that difficulties with long-term memory can be extended to, and may even be partly attributable to, basic problems with short-term memory and/or perception, such as our hippocampal patient's reported difficulties in discriminating between different views of spatially similar environments (e.g., different parts of a car park). This review addresses these questions by summarising novel findings from recent neuropsychological and neuroimaging investigations of memory and perception, in particular those that have utilised paradigms that allow one to directly contrast results in humans with those obtained from MTL lesion studies in animals.

\footnotetext{
1 In this transcript, our patient comments that her difficulties with remembering and navigating extend to both unfamiliar and familiar environments (a pattern contrary to some hippocampal cases, but not others, see Maguire et al., 2006; Rosenbaum et al., 2000, 2005; Teng and Squire, 1999). As the extent of our patient's difficulties with navigation have not been empirically tested, however, it is not possible to know if her self-report is an accurate representation of her navigational difficulties. See Spiers and Maguire (2007) for a detailed review of remote spatial memory after MTL damage.
}

These investigations are important in that they reveal significant convergence in the performance seen in humans and other animals, including differential sensitivity across MTL regions in processing and remembering of object and spatial information. This article concludes by (a) proposing a material-specific model of MTL function that highlights a role for this region in both memory and perception (emergent memory account, EMA), (b) asking whether it is possible to integrate these new findings into existing models of the MTL that focus on recollection and item-based recognition (Aggleton, Sanderson, \& Pearce, 2007; Brown \& Aggleton, 2001; Diana, Yonelinas, \& Ranganath, 2007; Eichenbaum, Yonelinas, \& Ranganath, 2007; Mayes, Montaldi, \& Migo, 2007), and (c) by broadening our account beyond object and spatial processing to address questions about how retrieval of other types of contextual information (e.g., temporal) may require the hippocampus (Charles, Gaffan, \& Buckley, 2004; Kumaran \& Maguire, 2006).

As this review focuses on a relatively new account of how the MTL contributes to memory, we provide a brief description of EMA here, expanding on this account at the end of the article. EMA proposes that memory is the outcome of a dynamic interplay between hierarchically organised perceptual representations distributed throughout the brain (see also Buckley \& Gaffan, 2006; Bussey \& Saksida, 2005; Bussey, Saksida, \& Murray, 2005; Murray, Saksida, \& Bussey, 2007) and prefrontal cortex, which supports flexible and adaptive processing necessary for unpredictable task demands (Duncan, 2001; Gaffan, 1994; Parker \& Gaffan, 1998; Wise, Murray, \& Gerfen, 1996). In this view, MTL regions do not merely act as a repository for long-term episodic memory traces of activations in non-MTL regions involved in perception, but instead function as active players that create complex conjunctive object and spatial representations critical for both accurate perception, and as a result, for accurate memory (O'Neil, Cate, \& Kohler, 2009). Consequently, memory deficits arise after MTL injury due to an inability to form new, and to access previously stored, conjunctive representations of objects and scenes that are differentially dependent upon the perirhinal cortex and hippocampus, respectively. EMA predicts that deficits after MTL damage should be modulated by material type and extend to long-term memory, short-term and implicit memory, as well as perceptual discrimination of complex, conjunctive, objects and scenes. We review evidence in favour and against this account below, starting with a brief historical description of the role of the MTL in long-term memory.

\subsection{The medial temporal lobe and long-term memory}

Since the seminal publications by Scoville and Milner (Scoville, 1954; Scoville \& Milner, 1957), which reported severe amnesia as a consequence of surgical removal of sections of the MTL bilaterally, it has been clearly demonstrated that structures in the MTL are critically involved in some, but not all, aspects of human memory. A key division that emerged within the first three decades of amnesia research was between impaired long-term declarative memory (our conscious memory for facts and events) and preserved nondeclarative memory (unconscious improvements in performance). Amnesic individuals showed poor conscious recall of word lists, pictures and recently experienced episodic events and semantic facts, while demonstrating unconscious improvements in performance (i.e., better accuracy or faster reaction times) after studying words or pictures and performing novel motor actions (Corkin, 1968; Keane, Gabrieli, Mapstone, Johnson, \& Corkin, 1995; Knowlton \& Squire, 1993; Manns \& Squire, 2001; Schacter, Chiu, \& Ochsner, 1993). Similarly, studies highlighted a dissociation in amnesic individuals between long-term and short-term memory, with normal immediate repetition of unfamiliar sequences of numbers (digit span), but impaired learning and memory for new material over 
longer delays (Damasio, Eslinger, Damasio, Van Hoesen, \& Cornell, 1985; Sidman, Stoddard, \& Mohr, 1968).

In terms of the anatomical structures that support declarative memory, it was initially assumed that the hippocampus was key to this type of long-term memory as it was frequently affected in amnesia (although see Correll \& Scoville, 1965a, 1965b; DeJong, 1973; Scoville \& Milner, 1957). A major development in the field, however, was the discovery that other structures in the MTL, such as the perirhinal cortex and entorhinal cortices, also make key contributions to memory (Murray \& Mishkin, 1986; see also Meunier, Bachevalier, Mishkin, \& Murray, 1993; Suzuki, ZolaMorgan, Squire, \& Amaral, 1993). Consistent with these results, MRI scans in amnesic individuals, such as the famous amnesic patient HM, revealed more extensive damage involving the hippocampal complex and entorhinal and ventral perirhinal cortices (Corkin, Amaral, Gonzalez, Johnson, \& Hyman, 1997). Consequently, it became generally accepted that structures within the MTL (e.g., the hippocampal complex, entorhinal cortex, perirhinal cortex and parahippocampal cortex) encompassed a single system supporting declarative memory, with similar contributions made by each of these structures to memory (Manns, Hopkins, Reed, Kitchener, \& Squire, 2003; Squire, 1982; Squire \& Alvarez, 1995; Squire, Stark, \& Clark, 2004; Squire, Wixted, \& Clark, 2007; Squire \& Zola-Morgan, 1991). The role played by the MTL in declarative memory was presumed to be temporary, with amnesic patients typically revealing better recall of memories from the remote compared to the recent past (Bayley, Hopkins, \& Squire, 2003; Bright et al., 2006; although see Moscovitch, Nadel, Winocur, Gilboa, \& Rosenbaum, 2006; Rosenbaum et al., 2008 for a different conceptualisation of the role of MTL structures in remote memory). A critical prediction of this view is that the degree of damage to MTL structures would correlate positively with the extent of memory impairment, a point that is discussed in more detail later in this review (Manns et al., 2003; Squire, 1982; Squire \& Alvarez, 1995; Squire \& Zola-Morgan, 1991; Squire et al., 2004).

\subsection{Functional specialisation within the MTL?}

In contrast to this traditional view, it has been suggested that different MTL areas may subserve distinct mnemonic processes. For example, Aggleton and Brown (1999) reviewed the literature on lesion, electrophysiological and early gene imaging studies in animals, alongside investigations of human amnesia, and proposed two independent anatomical networks supporting memory (see Fig. 1). The hippocampal-diencephalic system, including the

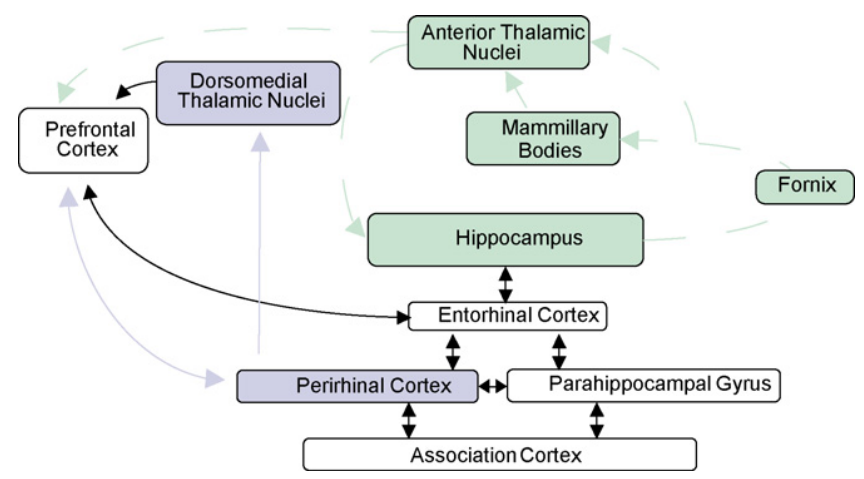

Fig. 1. Diagram showing how the different anatomical regions implicated in longterm declarative memory, in particular medial temporal lobe structures, may differentially support recollection (green boxes and arrows) and familiarity (purple boxes and arrows). Note, while a link between dorsomedial thalamus and perirhinal is well established neuroanatomically, there is currently little clinical evidence to support a clear role for this link in familiarity (see Aggleton \& Brown, 2006, for more discussion). (Please refer to the web version for the colour information.) hippocampus, fornix, mamillary bodies and anterior thalamus, is considered critical for the encoding and recall of episodic information, including spatial and temporal context, but is not necessary for familiarity-based item memory, in which there is a sense of knowing that an item has been seen previously in the absence of contextual information about where or when that item was encountered (Aggleton \& Brown, 2006; Aggleton, Hunt, \& Rawlins, 1986; Aggleton et al., 2007; Aggleton \& Shaw, 1996; Carlesimo et al., 2007; Mumby, Mana, Pinel, David, \& Banks, 1995; Murray, 1996; Tsivilis et al., 2008). By contrast, simple item recognition memory can be adequately supported by familiarity judgements, which are thought to be mediated by non-hippocampal MTL regions, including the perirhinal cortex and medial dorsal thalamic nuclei (Aggleton et al., 1986; Brown \& Aggleton, 2001; Brown, Wilson, \& Riches, 1987; Brown \& Xiang, 1998; Eacott \& Gaffan, 2005; Ennaceur \& Aggleton, 1997; Ennaceur, Neave, \& Aggleton, 1996; Gaffan, 1994; Gardiner, 1988; Holdstock, Shaw, \& Aggleton, 1995; McMackin, Cockburn, Anslow, \& Gaffan, 1995; Mishkin, Suzuki, Gadian, \& Vargha-Khadem, 1997; Sauvage, Fortin, Owens, Yonelinas, \& Eichenbaum, 2008; Zhu, Brown, McCabe, \& Aggleton, 1995; Zhu, McCabe, Aggleton, \& Brown, 1996; Zhu, McCabe, Aggleton, \& Brown, 1997).

In support of this view, some amnesic patients show preservation of item memory after hippocampal damage (Aggleton \& Shaw, 1996; Aggleton et al., 2005; Baddeley, Vargha-Khadem, \& Mishkin, 2001; Brandt, Gardiner, Vargha-Khadem, Baddeley, \& Mishkin, 2008; Giovanello \& Verfaellie, 2001; Holdstock et al., 2002; Mayes, Holdstock, Isaac, Hunkin, \& Roberts, 2002; Tsivilis et al., 2008; Yonelinas et al., 2002), although there are notable exceptions (see Gold, Hopkins, \& Squire, 2006; Hamann \& Squire, 1997; Hirst et al., 1986; Hirst, Johnson, Phelps, \& Volpe, 1988; Manns et al., 2003; Reed \& Squire, 1997; Stark, Bayley, \& Squire, 2002; Wais, Wixted, Hopkins, \& Squire, 2006). The opposite pattern, impaired familiarity in the context of preserved recollection, has also been reported (Bowles et al., 2007; Simons et al., 2002). Similarly, functional neuroimaging studies in healthy participants have also revealed differential patterns of encoding activity in the hippocampus and perirhinal cortex, with the former predictive of subsequent source recollection but uncorrelated with item recognition, and the latter associated with item/familiarity-based recognition, but not subsequent source memory (Davachi, Mitchell, \& Wagner, 2003; Eldridge, Knowlton, Furmanski, Bookheimer, \& Engel, 2000; Kensinger \& Schacter, 2006; Ranganath et al., 2004; Viskontas, Carr, Engel, \& Knowlton, 2009; although see Gold et al., 2006; Kirchhoff, Wagner, Maril, \& Stern, 2000; Stark \& Okado, 2003).

A complementary account of MTL function is relational memory theory, which proposes that the hippocampus is involved in the rapid learning of associations between individual items and their associated context or event (Cohen \& Eichenbaum, 1993; Eichenbaum, 2004; Eichenbaum et al., 2007; Eichenbaum, Otto, \& Cohen, 1994; Moses \& Ryan, 2006). This is thought to allow episodic experiences to form relational networks that support episodic retrieval (Davachi \& Wagner, 2002; Eichenbaum, 2000a, Eichenbaum, 2000b; Eichenbaum, Dudchenko, Wood, Shapiro, \& Tanila, 1999; Eichenbaum et al., 1994; Fortin, Agster, \& Eichenbaum, 2002). By contrast, structures in the parahippocampal region, particularly perirhinal cortex, are thought to support memory for individual objects (Cohen, Poldrack, \& Eichenbaum, 1997; Cohen et al., 1999; Eichenbaum \& Cohen, 2001; Ryan, Althoff, Whitlow, \& Cohen, 2000).

Consistent with this view, Giovanello, Verfaellie, \& Keane (2003) found that amnesic individuals showed poor memory for associations among items when item recognition performance was equated to that of controls (see also Turriziani, Fadda, Caltagirone, \& Carlesimo, 2004; although see Stark et al., 2002). In a sub- 
sequent functional neuroimaging study in healthy participants, hippocampal activation was associated with the requirement for relational or associative processing at retrieval (Giovanello, Schnyer, \& Verfaellie, 2004). Similarly, in an encoding study Davachi and Wagner (2002; see also Mitchell, Johnson, Raye, \& D'Esposito, 2000) showed that the hippocampus was activated to a greater extent by relational processing (i.e., reordering a triplet of words on the basis of their desirability) than it was during item-based processing (i.e., repeating the words in the order they were presented). Bilateral posterior parahippocampus and right entorhinal cortex, however, showed the opposite pattern of activity, with a greater increase during simple repetition compared to reordering.

A related model, termed 'binding of item and context (BIC), has recently been proposed (Diana et al., 2007; Eichenbaum et al., 2007). In this model, three MTL brain regions (perirhinal cortex, hippocampus and the posterior parahippocampal gyrus) are proposed to work together to support different aspects of recognition memory. The perirhinal cortex is thought to be critical for encoding and retrieving information about items to be remembered, while the posterior parahippocampal cortex stores information about the context (both spatial and non-spatial) in which items are presented. Binding of these two critical pieces of information is supported by the hippocampus (item-context associations). Notably, unlike other models of MTL function that highlight the key role of the hippocampus in recollection, the BIC model allows for recollection to be supported by perirhinal and/or parahippocampal cortices when the retrieval task involves re-instantiation of either the item and/or context information, respectively (e.g., recollection for item-item associations may result in increased activity in perirhinal cortex (in addition to the hippocampus), and increased activity may be seen in parahippocampal cortex (in addition to the hippocampus) if context is re-instantiated at retrieval, see Mayes et al., 2007, for further discussion of the role of the MTL in associative memory). As contextual information is not necessary to make a familiarity judgment, which can be supported solely by item representations, familiarity processes are considered the domain of perirhinal cortex, consistent with other accounts of MTL function (Brown \& Aggleton, 2001; Mayes et al., 2007).

Proponents of a unitary account of MTL function have challenged these views, in particular the assumption that the hippocampus and perirhinal cortex are differentially involved in recollection and familiarity, respectively (Kirwan, Wixted, \& Squire, 2008; Squire et al., 2007; Wixted, 2007). Instead, these authors propose that many studies contrasting recollection and familiarity have confounded memory strength. They argue that confidence is often higher for items that are associated with correct source judgements than for items that are associated with incorrect source judgements, and consequently the comparison of recollection versus familiarity is confounded by the contrast of strong versus weaker memories. There is significant debate in the literature about whether memory strength can explain dissociations in recollection and familiarity (see Diana, Yonelinas, \& Ranganath, 2009); notably, while proponents of the memory strength view argue that the MTL is not easily fractionated, especially along the lines of recollection and familiarity, they are open to the possibility that the perirhinal cortex and the hippocampus may not have the same function. For example, Squire and colleagues note that there may be differences in how these structures code information, with perirhinal cortex more stimulusspecific in its processing and the hippocampus more abstract, perhaps coding conjunctions of the broad range of attributes that comprise an episodic memory (a proposal that seems to be edging closer towards a functional specialisation account, particularly one in which information and/or material may be important, Bird \& Burgess, 2008a; Diana et al., 2009; Graham, Lee, \& Barense, 2007).

\section{Going beyond declarative memory?}

Despite differences in the way these theories conceptualise the contribution of MTL structures to memory, the accounts described above have in common the fact that they restrict the role of structures in the MTL to long-term declarative memory (e.g., encoding and retrieval of a memory trace, whether this is of an item, context or the association between an item and context). This tenet-which for decades has been a cornerstone of many accounts of human memory-has recently been challenged. While it is undisputed that the MTL is involved in memory, and that damage to this structure results in significant memory impairment, studies in animals and humans have also revealed visual discrimination deficits that cannot easily be accounted for by an impairment in declarative memory (Bartko, Winters, Cowell, Saksida, \& Bussey, 2007a, 2007b; Buckley, Booth, Rolls, \& Gaffan, 2001; Buckley \& Gaffan, 1997, Buckley \& Gaffan, 1998b; Buckley, Gaffan, \& Murray, 1997; Bussey, Saksida, \& Murray, 2002, 2003; Eacott, Gaffan, \& Murray, 1994; Graham et al., 2006; Lee, Buckley, et al., 2005; although see Hampton \& Murray, 2002). The findings from animals have been reviewed extensively elsewhere (e.g., Aggleton \& Brown, 2005; Baxter, 2009; Buckley \& Gaffan, 2006; Bussey \& Saksida, 2005, 2007; Bussey et al., 2005; Gaffan, 2001, 2002; Graham et al., 2007; Hampton, 2005; Lee, Barense, \& Graham, 2005; Murray \& Bussey, 1999; Murray et al., 2007; Suzuki, 2009), so the following sections are restricted to a brief description of the animal experiments that set the foundation for the studies in humans (e.g., concurrent discrimination (Bussey et al., 2002) and oddity judgement (Buckley et al., 2001)).

\subsection{Visual discrimination}

In a series of recent papers, Bussey and colleagues (Bussey \& Saksida, 2007; Murray \& Bussey, 1999; Murray et al., 2007; see also Buckley \& Gaffan, 2006) have proposed an alternative account for the role of MTL structures, particularly perirhinal cortex, in both perception and memory. Highlighting anatomical links with other regions in the visual ventral processing stream, Bussey and Saksida (2007) propose that perirhinal cortex is the apex of this stream, responsible for processing and storing representations of complex feature conjunctions. These representations help to resolve "feature ambiguity", a property of visual discrimination that arises when object features are part of both a rewarded and a non-rewarded object, both in perceptual and memory tasks. To test this proposal, monkeys with perirhinal lesions learned to discriminate between pairs of stimuli over a series of repeated trials (Bussey et al., 2002). In a minimum feature ambiguity condition there was no overlap between the features that comprised the rewarded and non-rewarded stimuli, whereas in a maximum feature ambiguity condition all features were present in both the rewarded and non-rewarded item (see Fig. 2a). Solving the maximum ambiguity task, therefore, required the animal to integrate multiple object features in order to successfully distinguish between items. In an intermediate condition, one feature was shared across the rewarded and non-rewarded items. Strikingly, the authors found that the monkeys with perirhinal lesions performed normally on the minimum feature ambiguity condition, but were impaired at both the intermediate and maximum conditions (see Fig. 2a and c). These deficits were subsequently extended to single-pair discriminations: monkeys with perirhinal lesions were impaired at discriminating morphed pairs of stimuli, particularly when feature ambiguity was high, as measured by the degree of morphing between the pairs (Bussey, Saksida, \& Murray, 2003). Notably, monkeys with hippocampal lesions performed normally on the same tasks (Saksida, Bussey, Buckmaster, \& Murray, 2006). 
(A)

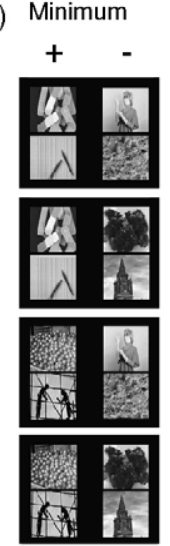

Intermediate

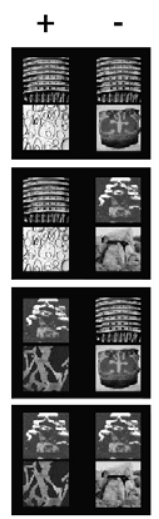

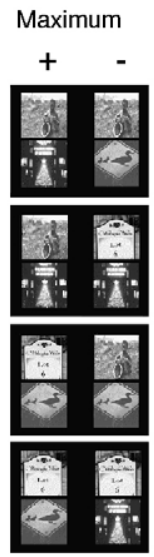

(B)

Minimum

$+$
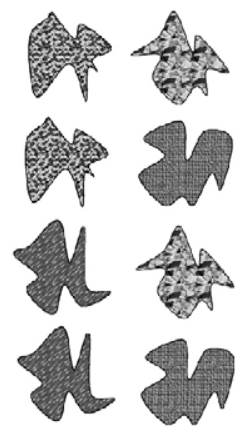
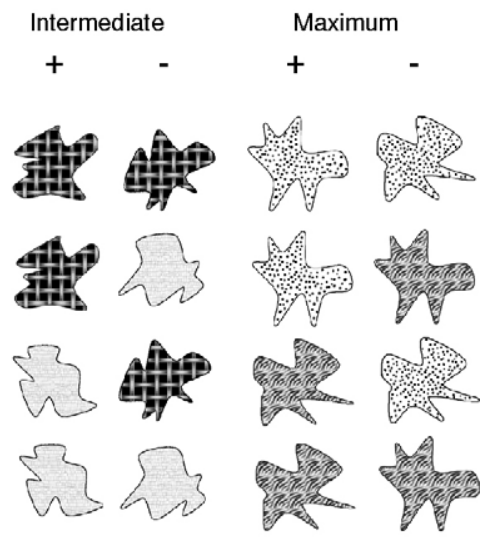

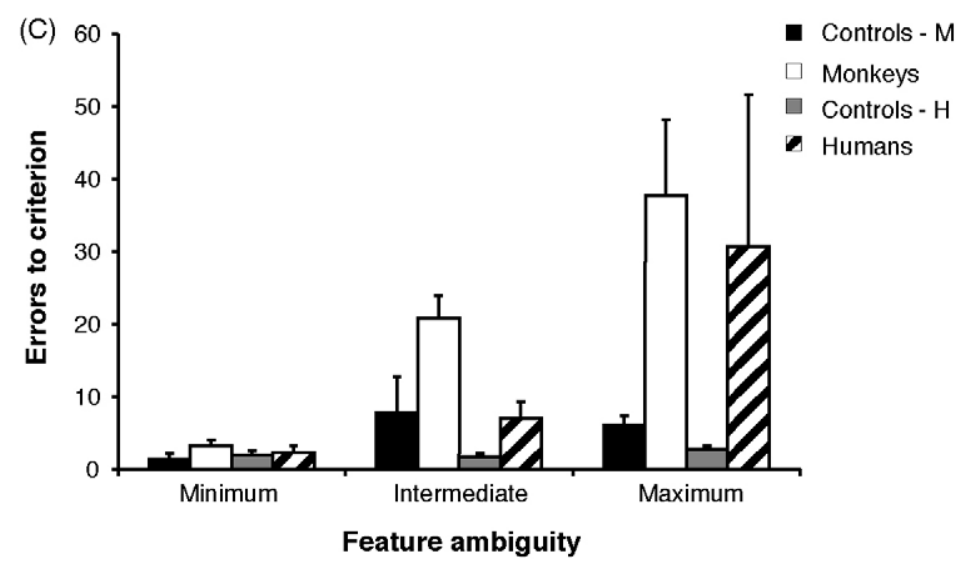

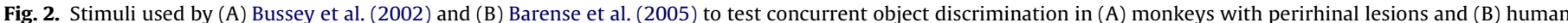

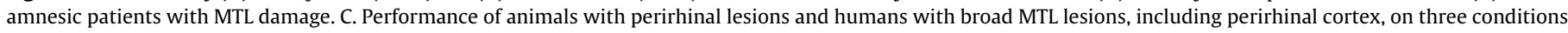

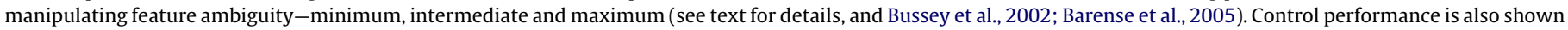
for both groups (controls-M = control monkeys; controls- $\mathrm{H}$ - healthy human participants matched to the amnesic individuals).

These findings, along with a series of complementary studies (Bartko et al., 2007a; Bussey et al., 2003; Cowell, Bussey, \& Saksida, 2006; Winters \& Bussey, 2005), imply that perirhinal cortex is critically involved in analysing and representing complex conjunctions of the features that comprise an individual object (see Bussey \& Saksida, 2005, 2007, Winters, Saksida, \& Bussey, 2008 for review). The presence of feature ambiguity in a task involving learning or visual discrimination forces the animal to integrate multiple object features in order to successfully distinguish between items (configural object processing). By contrast, simple object features, such as colour, are thought to be dependent upon modality-specific regions lateral and posterior to the perirhinal cortex (such as V4 and area TE/TEO, Buckley et al., 1997), a proposal consistent with the finding that performance on difficult colour and size discriminations is normal in perirhinal cortex lesioned monkeys (Buckley et al., 2001; Bussey et al., 2003).

A recent study by Barense et al. (2005) tested human participants with focal lesions to the MTL on modifications of the same concurrent discrimination task used in monkeys (Bussey et al., 2002; Saksida et al., 2006). Four new conditions were developed-barcodes, blobs, bugs and beasts-in which feature ambiguity was systematically manipulated in exactly the same way as in the monkey experiment (see Fig. 2b for an example of the blob stimuli). Two patient groups were contrasted: individuals with involvement of the hippocampus, and participants with broader MTL lesions affecting the hippocampus and non-hippocampal structures, including the perirhinal cortex (see Fig. 3a and b). Consistent with findings in monkeys, patients with hippocampal damage showed no impairment in any of the four conditions, even at the highest level of feature ambiguity (maximum condition) or on especially difficult discriminations (barcodes). Patients with broader lesions including perirhinal cortex were impaired, however, but only when feature ambiguity was stressed (i.e., in the intermediate and maximum ambiguity conditions). Performance was virtually normal on all four conditions of minimum ambiguity (see Fig. 2c). This evidence suggests that the human perirhinal cortex, but not the hippocampus, is critically involved in processing conjunctions of object features (although see discussion re lesion size later in the article). Moreover, a perceptual factor (feature ambiguity), not memory load (the number of items to remember was held constant across all conditions), was the critical factor in determining involvement of perirhinal cortex.

It is important to note that this task confounds learning and perception; for example, if, as argued by Hampton (2005), discriminations that are higher in feature ambiguity place a greater strain on learning, then the effect of feature ambiguity on performance would be predicted by accounts of perirhinal cortex that propose a selective involvement for this structure in memory. There are a number of reasons why this is unlikely. First, control performance was matched across all ambiguity conditions, indicating that healthy controls did not find learning the maximum ambiguity conditions to be more difficult. Second, amnesic participants with hippocampal damage performed completely normally on all 11 conditions (even when tested on very difficult maximum ambiguity barcodes), despite showing equivalent impairment in recall-based memory to the patients with broader MTL lesions (see also Saksida et al., 2006). Under a unitary account of MTL function (Squire et al., 2007), it might be expected that selective lesions to the hip- 

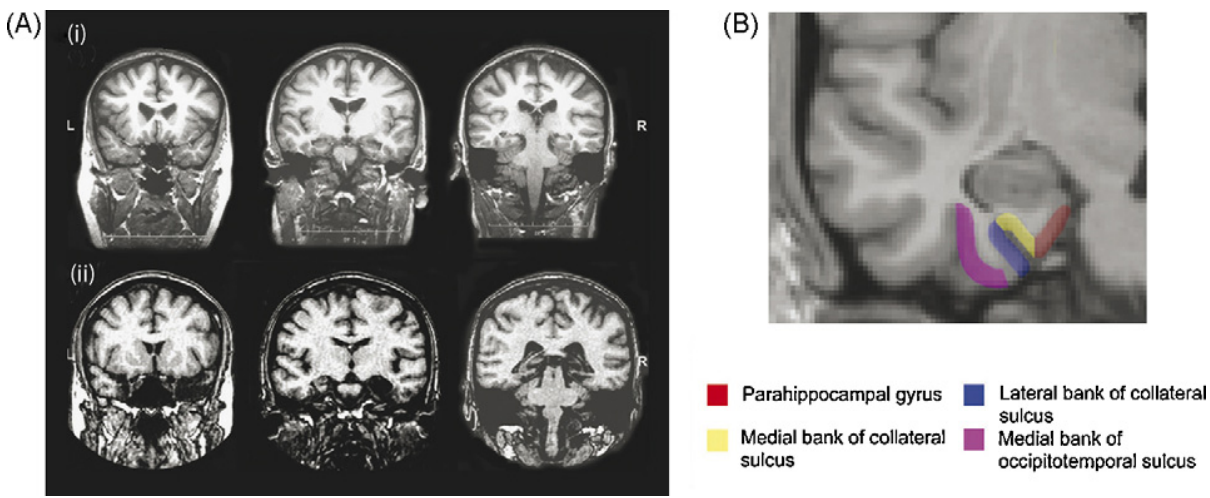

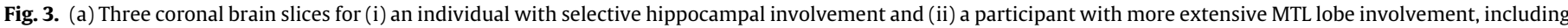

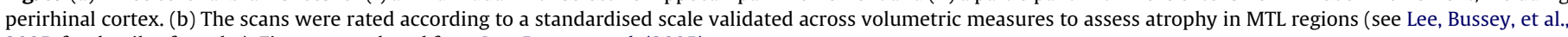
2005, for details of results). Figure reproduced from Lee, Bussey, et al. (2005).

pocampus would also result in impaired performance if a deficit in learning were the primary cause of the pattern seen in the MTL cases.

Another investigation using visual discrimination extended the conclusions that could be drawn from Barense et al. (2005) by comparing performance on face, scene, object and colour discrimination. This experiment investigated whether the hippocampus, which most memory models consider to play a key role in memory for contextual information (of which spatial relationships can be considered one type, Aggleton \& Brown, 1999; Diana et al., 2007; Eichenbaum et al., 2007), was necessary to discriminate between complex scenes with high levels of "spatial ambiguity" (e.g., two scenes with many spatial features in common). Participants were asked to indicate which of two items was the most similar to a target stimulus (Lee, Bussey, et al., 2005). The pairs to be discriminated were created by morphing two prototype images (one of which was the target stimulus) to create trial unique pairs, in which the pairs were blended either $0-9 \%, 10-19 \%, 20-29 \%, 30-39 \%$, or $40-49 \%$. In some conditions, therefore, the pairs had a large number of features in common (high feature ambiguity) making it more difficult to determine which item matched the original stimulus. In other pairs, the stimuli were quite distinct, and could easily be solved on the basis of a single feature (low feature ambiguity). Participants were tested in two conditions: (a) discrimination when the prototype target was shown for a series of initial learning trials at the beginning of the experiment, but not during test trials (target absent) and (b) when the prototype image was presented above each set of test trials allowing direct matching (target present). Different categories of stimuli were tested, thus allowing the assessment of the role of different MTL structures in processing faces, objects and scenes with varying levels of ambiguity (both feature and spatial). The same two groups of patients as used in Barense et al. (2005) were contrasted: individuals with hippocampal damage, and cases with more extensive lesions, including to the hippocampus and perirhinal cortex (see Fig. 3a).

In support of the idea that the human hippocampus may be involved in scene perception, both patient groups were impaired in the discrimination of high ambiguity spatial scenes regardless of whether the target image was present or absent. By contrast, the MTL group, but not the hippocampal group, demonstrated an additional impairment in discriminating high ambiguity objects and faces, suggesting a role for the perirhinal cortex in processing complex conjunctions of object features (see Fig. 4 for data from the target present condition). Notably, object discrimination deficits in the MTL participants were less robust on this task than face discrimination impairments (see also Levy, Shrager, \& Squire, 2005, and Shrager, Gold, Hopkins, \& Squire, 2006, discussed below), with a small impairment in the condition where the target was absent, but less consistent effects (deficits in only 2/3 individuals with MTL lesions) when the target was continually present. Importantly, neither patient group had any difficulties discriminating between blended colour patches, indicating that the patients had a selective difficulty in discriminating conjunctions of features, but were normal at single feature discriminations. Consistent with the hypothesis that 'ambiguity' was an important factor in performance, both for objects/faces and scenes, the patients made increasing errors as the perceptual similarity between the pairs increased. These findings extended the conclusions that can be drawn from the animal studies by highlighting different roles for the perirhinal cortex and hippocampus in object and scene discrimination, respectively. In the case of the perirhinal cortex, this role was restricted to the representation of features that comprise complex object and face stimuli, whereas in the hippocampus, it involved representing the unique relationships between objects that comprised a scene (see Bussey \& Saksida, 2007, for a similar proposal).

Two subsequent studies have failed to replicate these findings (Levy et al., 2005; Shrager et al., 2006). This has led to criticism of the original Lee, Bussey, et al. (2005) study, with the suggestion that the deficits emerged because controls, but not patients, benefited from learning across trials, especially in situations where non-trial unique stimuli were used, or when trial-unique stimuli were generated from the same prototype images (as in Lee, Bussey, et al., 2005). In Levy et al. (2005), two patients (EP and GP) with

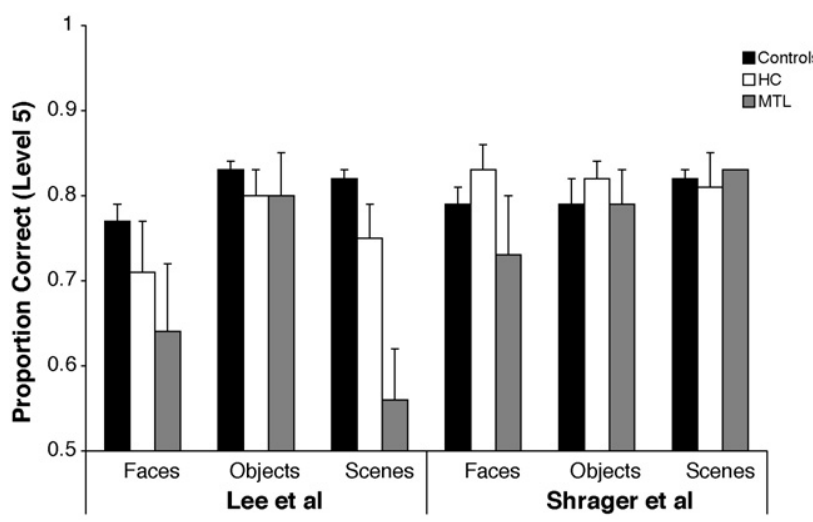

Fig. 4. Performance of three groups of participants (HC: individuals with selective hippocampal damage; MTL: cases with more extensive lesions to the MTL, including to perirhinal cortex; and matched controls) on simultaneous visual discrimination for blended faces, objects and scenes. Data from Level 5 (40-49\% blend), Lee, Barense et al. (2005), Lee, Buckley, et al. (2005), Lee, Bussey, et al. (2005) and Shrager et al. (2006). 
lesions extending throughout the MTL and including perirhinal cortex, were tested on three experiments in which the participants were asked to indicate which of a pair of blended pictures was closest to a target. Similar to Lee, Bussey, et al. (2005), the patients were tested in two conditions (target absent and target present). In the latter condition, both patients performed normally, while in the former - when the target was not present on the screen - one case (EP) was clearly impaired. EP also showed poor discrimination in a third experiment in which he was required to indicate whether a pair of blended images were the same or different (the target was not present).

In a further investigation, Shrager et al.(2006) tested two groups of patients (cases with hippocampal damage and two individuals with MTL damage involving perirhinal cortex) on object and face discrimination tasks, again contrasting a condition in which a target was absent with a condition in which the target was present. An additional experiment extended the paradigm by using unique prototype stimuli in each trial in the simultaneous (target present) condition. Whereas the hippocampal group performed normally on all conditions, one out of two of the MTL cases with perirhinal lesions, EP, showed difficulties with face discrimination, performing at the bottom of the control range (regardless of whether the target was present or absent, see Fig. 4). On the trial-unique prototype version of the task, however, there were no significant differences in performance between control participants, individuals with selective hippocampal lesions, and patients with more extensive MTL damage. It should be noted, however, that despite this lack of a main effect of group, there was some indication that the two patients with broader MTL involvement did not show similar profiles of performance. Whereas one patient was most likely impaired, the other performed well, a pattern reducing the likelihood of obtaining a statistically significant effect. Shrager et al. (2006) also investigated scene discrimination of morphed landscapes using both repeated stimuli and trial-unique scenes. In contrast to Lee, Bussey, et al. (2005), the MTL-lesioned patients performed well, even when they were required to remember stimuli across multiple trials. Inspection of the experimental stimuli used by Shrager et al., however, reveals the presence of readily identifiable single features in one or both of the target images that could provide an opportunity for easy identification of the blended images. The stimuli used in these experiments, therefore, may have been unlikely to stress conjunctive processing of features dependent upon MTL regions.

Taken together, Levy et al. (2005) and Shrager et al. (2006) imply that impairments in object discrimination are most likely to be elicited when participants are required to remember the target image throughout the task or when the images used in the experiment are repeated across trials (e.g., when trial-unique modifications of the same prototype stimuli, rather than unique prototypes, are presented for each trial). This explanation is consistent with an inability to remember the target item during the test (in amnesics) and/or a beneficial effect of repetition from the use of the same prototype stimuli (in controls), suggesting an impact of declarative memory on performance rather than a deficit in visual perception per se. Inconsistent with this proposal, analyses of task performance across the five trial blocks in each condition (both for target present and absent) in Lee, Bussey, et al. (2005) revealed no significant effects of learning (in controls and patients), thus countering the argument that the patients might have forgotten the target image in the target absent condition, and that learning by the controls, but not amnesic individuals, might explain the observed impairments (Lee, Bussey, et al., 2005). This discrepancy implies that there may be other key differences across tasks and/or patients, as well as weaknesses in particular methods, such as using morphing techniques to create blended images for stimuli (see Baxter (2009), Squire, Shrager, \& Levy (2006), Suzuki (2009),
Suzuki and Baxter (2009), and discussions related to this issue later in the review).

\subsection{Oddity judgement}

In one of the most influential papers on this topic, Buckley et al. (2001) utilised an oddity judgement paradigm in which monkeys with perirhinal lesions were required to select the odd stimulus from an array of six images taken from a variety of stimulus categories (see Fig. 5). Whereas some of these discriminations could be made on the basis of single features (e.g., oddity judgement for shape, colour, and size), other discriminations placed an increased demand on perceiving conjunctions of features (e.g., oddity judgement for faces, and for objects masked with varying degrees of visual noise). Buckley and colleagues found that following perirhinal lesions, discrimination of simple single features was intact whereas more complex discriminations for faces and objects were impaired, particularly when different views of the stimuli were presented, see Fig. 5). An initial study in amnesic individuals, however, used a similar version of this task and failed to replicate these findings: patients with perirhinal cortex damage performed within the normal range across all stimulus conditions, even on tasks that were sensitive to perirhinal cortex damage in monkeys (Stark \& Squire, 2000). Notably, however, closer inspection of the results again hints at a difficulty with face oddity-with two out of three individuals with perirhinal lesions showing poorer performance than controls (see also the poor performance of EP on this task, z-score $=-3.59$, reported in Levy et al., 2005), suggesting that performance on oddity judgement may not always be normal after MTL injury.

A criticism of the original Buckley et al. (2001) oddity study is that performance in animals was assessed by trials to criterion (monkeys had to reach a criterion of $\geq 90 \%$ correct, see Hampton, 2005), which may be more of a measure of learning rather than perception. In a more detailed discussion of this issue, Hampton (2005) challenges a number of the conclusions drawn from the animal studies on this topic (Buckley \& Gaffan, 1997; Buckley \& Gaffan, 1998a, Buckley \& Gaffan, 1998b; Buckley et al., 2001; Bussey et al., 2002, 2003), in particular (a) focusing on the lack of evidence for significant changes in the magnitude of deficits in relearning visual discriminations compared to initial learning and (b) noting that many of the dependent measures reported by Bussey, Buckley and colleagues were of learning rather than of perception (although see Baxter (2009), Buckley (2005) and Bussey et al. (2005) for responses to these criticisms).

To address these concerns, Lee, Buckley, et al. (2005) report two experiments using oddity judgement in amnesic individuals with MTL damage (the same participants described by Barense et al., 2005; Lee, Bussey, et al., 2005). In the first experiment aimed at replicating findings across animals and humans - the same tasks and stimuli developed by Buckley et al. (2001), and subsequently used by Stark and Squire (2000), were given but with two significant changes: (a) the number of stimuli presented was increased (sets of twenty items were used, in comparison to the sets of ten used in Stark \& Squire, as early studies in animals found that larger set sizes were more likely to elicit impairment, presumably due to a resulting increase in feature ambiguity (Buckley \& Gaffan, 1997; Bussey et al., 2002; Eacott et al., 1994; although see Hampton, 2005), and (b) novel and familiar stimuli were considered separately, given the possibility that performance might be better when items are familiar (compared to novel), as the perceptual discrimination may benefit from top-down influences from semantic knowledge (see MacKay \& James, 2009, for evidence in support for this possibility). Apart from these changes, the paradigm was designed to be similar to that used by Buckley et al. (2001) and Stark and Squire (2000), and consequently involved the repetition of trials, thereby confounding perception and memory if controls-but 
(A)

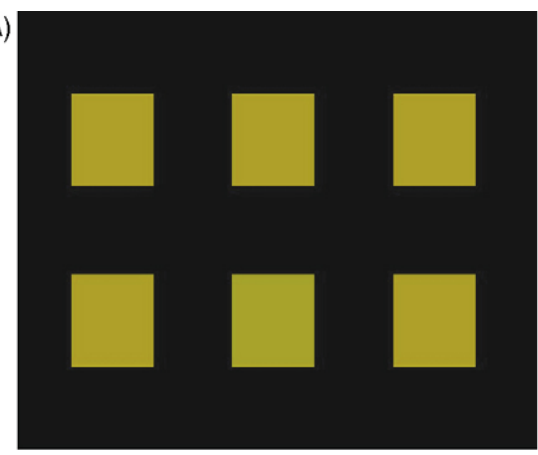

(C)

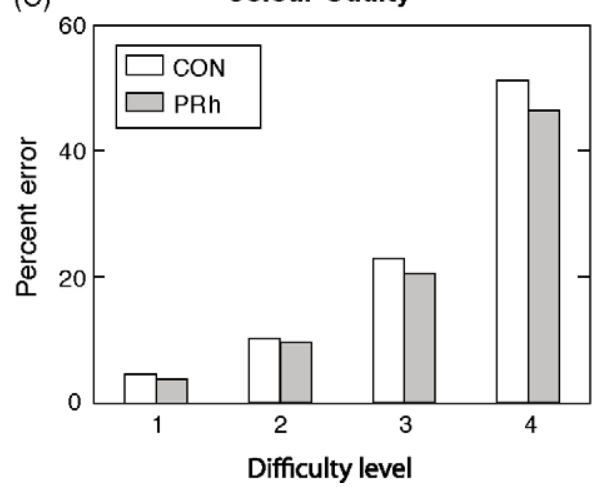

(B)
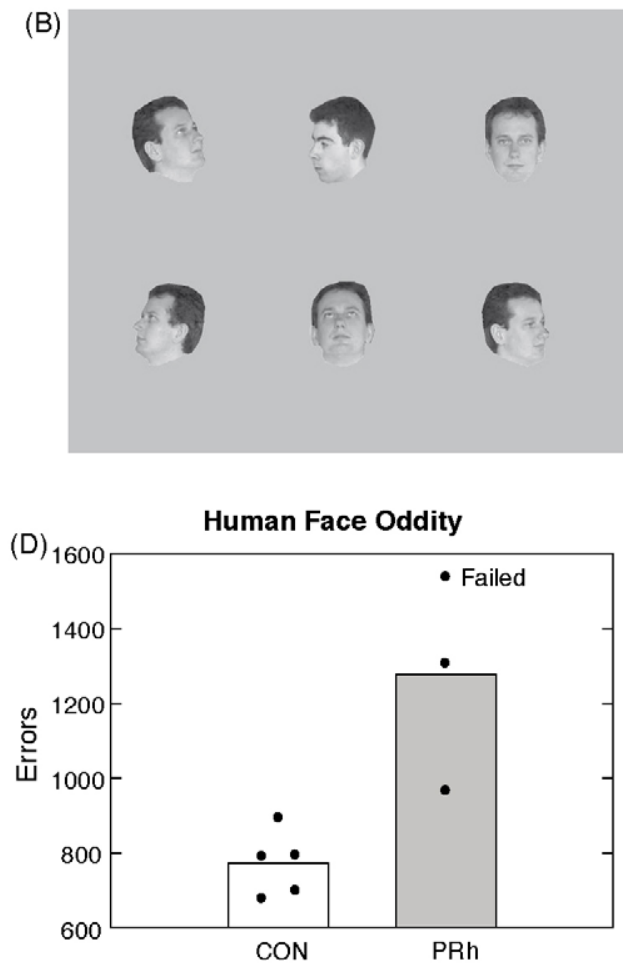

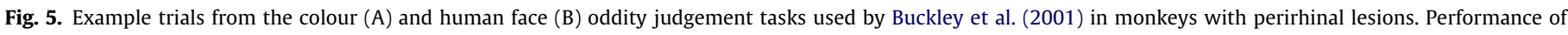

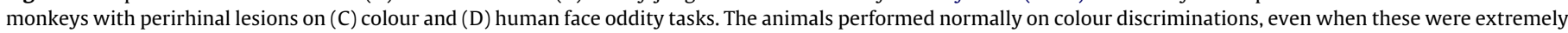
difficult, but were significantly impaired on face oddity.

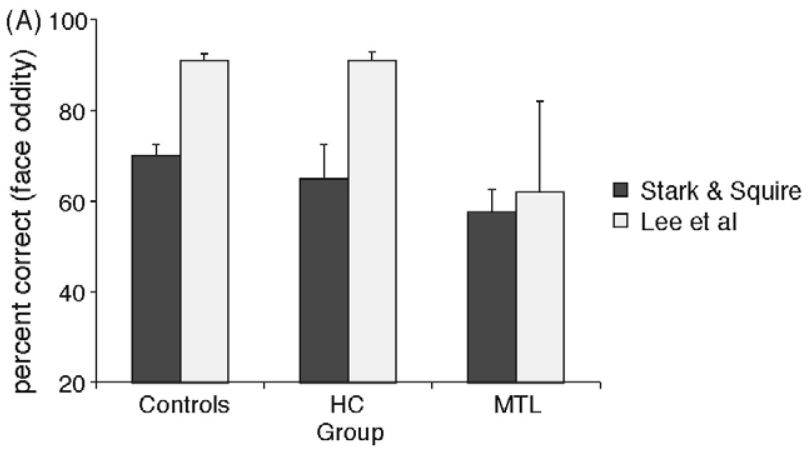

(B)

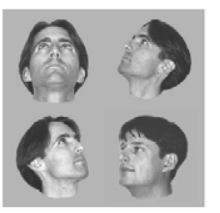

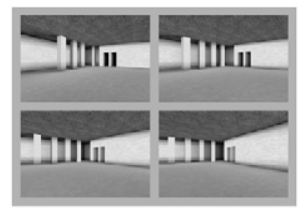

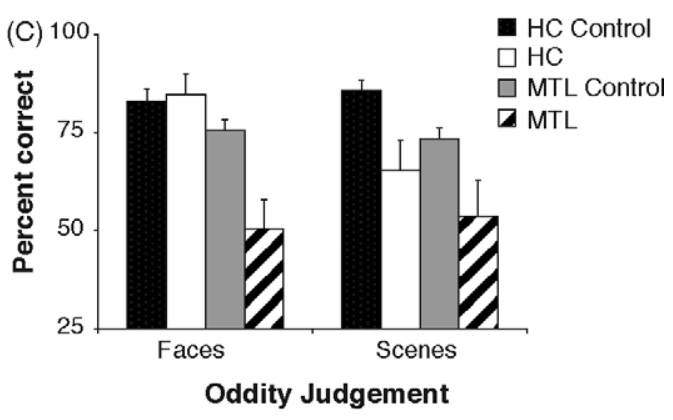

Fig. 6. A. Comparison of the performance of three groups of participants (HC: individuals with selective hippocampal damage; MTL: cases with more extensive lesions to the MTL, including to perirhinal cortex; and controls) on face oddity (data shown from Stark \& Squire, 2000 (estimated from original paper) and Lee, Buckley, et al., 2005, Experiment 1). B. Example stimuli from trial-unique face and scene oddity (Lee, Buckley, et al., 2005, Experiment 2). C. Performance of four groups of participants (HC patients, MTL patients, and their matched controls) on trial-unique face and scene oddity (Lee, Buckley, et al., 2005, Experiment 2). 
not patients-benefited from repeated presentation of trials (see Hampton, 2005). To overcome this potential confound, a second experiment investigated visual perception independent of learning by utilising a set of novel oddity tasks comprising trial-unique items. This test battery included face oddity, as well as a spatial version of the oddity task (using virtual reality room stimuli) designed to be sensitive to hippocampal function (see Fig. 6b). In both conditions, three different views of the same item (face or scene) were presented alongside a different face or scene. This task, therefore, addressed potential criticisms of the animal studies, and provided an opportunity to extend the findings into the spatial domain using a paradigm that did not confound memory and perception.

Consistent with Lee, Bussey, et al. (2005), patients with hippocampal damage showed no difficulties with face oddity judgement (see also Stark \& Squire, 2000), whereas individuals with broader lesions to the MTL were impaired. MTL patients showed impairments in novel object oddity, but not in the colour, size, shape or familiar object tasks. Fig. 6a shows a comparison of the data on faces from Stark and Squire (2000) and Lee, Buckley, et al. (2005) (Experiment 1), respectively. As demonstrated in Fig. 6a, the controls in Stark \& Squire performed more poorly than those reported in Lee, Buckley et al. Somewhat paradoxically, despite the increase in the number of items presented in Lee et al., the controls from this study performed approximately 20\% better than those reported in Stark and Squire (2000). Interestingly, the two patient groups made a similar number of errors. Consequently, the difference between these two studies seems to be attributable to differential performance across control groups, rather than significant differences in the performance of the patients per se.

In the trial-unique oddity task, Lee, Buckley, et al. (2005) found that patients with hippocampal damage, but no involvement of perirhinal cortex, were significantly impaired on oddity judgements for virtual reality rooms, but showed normal performance on oddity judgements involving faces. Cases with broader MTL lesions, including the hippocampus and perirhinal cortex, were also impaired on oddity judgement for virtual reality rooms, but additionally demonstrated significant deficits when they were required to make oddity judgements for faces (see Fig. 6c). Strikingly, the Lee, Buckley, et al. (2005) findings from both experiments, when considered alongside those of Buckley et al. (2001), confirm virtually identical profiles of performance on the original oddity tasks in perirhinal cortex lesioned monkeys and amnesic individuals with lesions including perirhinal cortex (e.g., preserved colour, shape, size and same view face oddity but impaired novel object and face oddity). The fact that the human participants show similar impairments on a non-trial unique and trial-unique face oddity paradigm addresses concerns about the design of the original tasks in monkeys (see Buckley, 2005; Hampton, 2005).

An intriguing aspect of the results from Lee, Buckley, et al. (2005) is the selective impairment demonstrated by the hippocampal patients on scene oddity judgement. This finding implies that the role of the hippocampus extends beyond contextual memory for the 'where' component of episodic memory to situations in which the perceptual analysis of scenes is required, even when there is no explicit requirement for long-term memory. Notably, this deficit was only evident when participants were asked to indicate the odd scene when it was presented alongside a set of three different views of another scene. Performance for same view scene oddity - the odd one out was shown with three identical views of another scene - was preserved in six out of seven patients. Although the scene deficit after hippocampal damage was only evident on different view discriminations, it seems unlikely that this can be attributed solely to an impairment in viewpoint independent processing, as the patients showed similar difficulties on the scene morph task in which viewpoint was irrelevant (Lee, Bussey, et al., 2005).

In a more recent experiment, Hartley et al. (2007) tested four patients with selective hippocampal damage on a simultaneous and delayed-match-to-sample ( $2 \mathrm{~s}$ delay) paradigm similar to Lee, Buckley, et al. (2005) oddity task. Participants were required to identify which of four scenes with similar topography matched a target (e.g., the scene was of the same place but from a different view). Although all four patients showed clear deficits in scene matching after a delay, only two of the four participants (one a
(A)
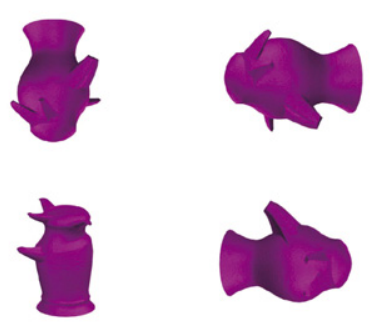

(C)

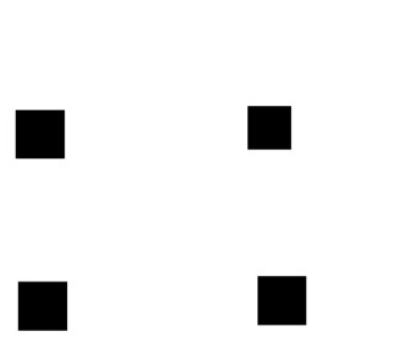

(B)
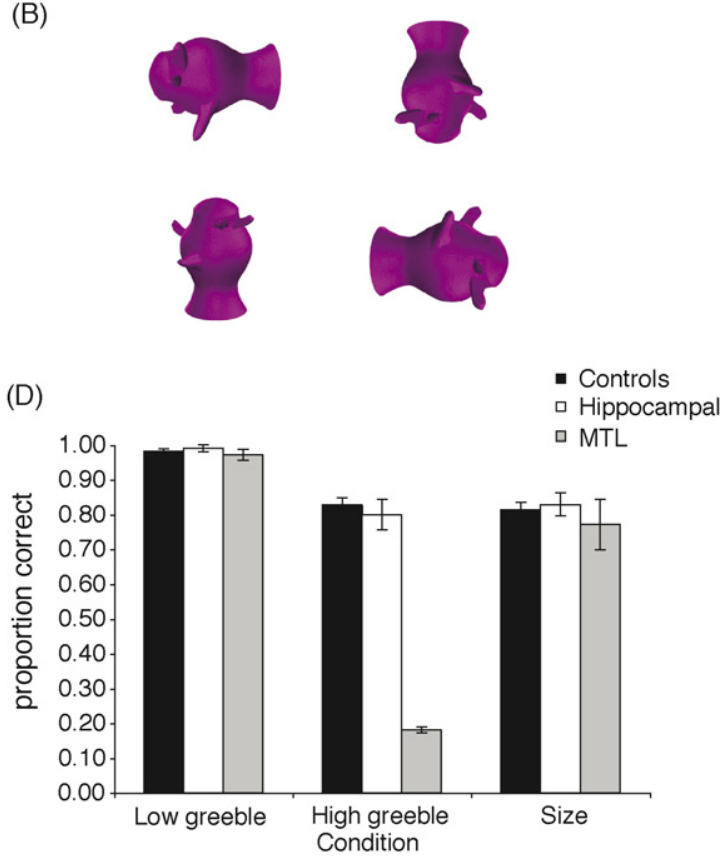

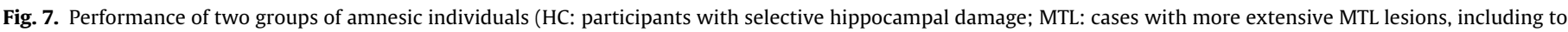

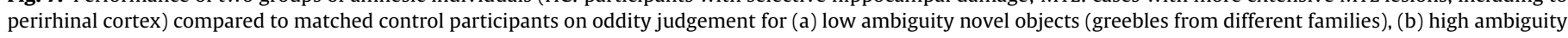
novel objects (greebles from the same family), and (c) size (Barense et al., 2007). 
case of developmental amnesia) were impaired on the simultaneous scene matching. This finding confirms-on a different set of stimuli and a different group of patients-that some amnesic individuals do show scene discrimination deficits, particularly for scenes that overlap in their spatial layout and relationships, but raises questions about why different patterns are observed across patients.

More recently, Barense, Gaffan, \& Graham (2007) designed two novel tasks to test the hypothesis that feature ambiguity was the critical factor influencing performance on object oddity. Amnesic patients with perirhinal cortex involvement showed striking deficits on trial-unique object oddity when items with a high number of overlapping features were presented. In one experiment, amnesic patients were asked to indicate which of seven 'fribbles' (object stimuli comprising four features that were systematically manipulated to create conditions of high and low ambiguity) was the odd one out. In a second experiment, oddity judgement for novel and familiar objects subjectively considered to have low and high ambiguity was contrasted. Barense et al. found severe deficits on both experiments in individuals with broad MTL lesions that included the perirhinal cortex, as long as feature ambiguity was high (see Fig. 7). When there was minimal overlap of features (in low ambiguity object conditions), or when single features (colour and size) were contrasted, patients with perirhinal lesions performed normally. This effect cannot be explained by task difficulty, as the size and colour control conditions were as difficult as the high ambiguity oddity tasks. Consistent with Lee, Buckley, et al. (2005) patients with hippocampal lesions showed no impairment on any condition. These findings are the first to demonstrate robust deficits in object processing beyond faces in patients with perirhinal involvement on a task for which there is no learning component, and highlight the importance of using complex object stimuli with overlapping features when testing the integrity of object processing and memory after perirhinal cortex damage.

\section{Convergent evidence from fMRI}

To explain the variability seen in their patients, Hartley et al. (2007) propose that impairments in simultaneous scene matching may arise only after combined hippocampal and parahippocampal damage, as opposed to more selective hippocampal involvement. Similarly, in the context of object processing and mnemonic deficits after MTL involvement, Shrager et al. (2006) have suggested that when patients show unpredicted patterns (e.g., they show impairment on a task that is not obviously declarative), they must possess structural and/or functional deficits outside the MTL (see also Suzuki (2009) for concerns relating to the anatomical damage in our patients). For example, a potential explanation for the difference between patient EP and GP on tests of face discrimination may be the fact that EP has greater damage to the fusiform gyrus than GP, thereby affecting regions known to be critical for face processing. The issue of structural damage in the amnesic patients discussed here, and reported elsewhere in the literature, is very important and will be discussed in more detail later (see section on outstanding questions). For now, however, this discrepancy highlights the importance of obtaining convergent fMRI evidence in healthy participants using the same tasks (see Baxter, 2009; Suzuki, 2009, for a similar plea).

In an fMRI experiment, therefore, Lee, Scahill, and Graham (2008) scanned healthy participants using the same trial-unique oddity tasks described in Lee, Buckley, et al. (2005) and found that, compared to face and size oddity conditions, oddity judgement for trial unique scenes resulted in increased activity in the posterior hippocampus and parahippocampal cortex (see Fig. 8a). In contrast, increased perirhinal cortex and anterior hippocampus activity was observed during trial-unique face oddity judgement (see Fig. 8b). One potential explanation for the activation patterns in the MTL is incidental episodic encoding. It is worth noting, however, that while posterior hippocampus and parahippocampal cortex showed significant modulation of activity over repetition of trials, this pattern was not seen in perirhinal cortex and anterior hippocampus. If the activation in perirhinal cortex for face oddity was associated with incidental encoding, activity in this region should have shown a statistically significant decrease with repetition(Henson, Cansino, Herron, Robb, \& Rugg, 2003).

Convergent evidence for the recruitment of perirhinal cortex during object oddity judgment has also been demonstrated using functional neuroimaging (Barense, Henson, Lee, \& Graham, 2009, see Fig. 8c; Devlin \& Price, 2007). For example, Devlin and Price (2007) found activation in perirhinal cortex bilaterally when participants performed difficult familiar object oddity judgements, but not when they performed simple object discriminations (e.g., spotting the cup when it was presented alongside three identical drills), or single feature discriminations (colour and shape oddity). Of particular interest in this study was evidence of a functional difference in activation between entorhinal and perirhinal cortex, with the former failing to show any stimulus (object vs. feature $($ colour $/$ size $) \times$ processing $($ easy/difficult) $)$ interaction, unlike perirhinal cortex.

Extending the findings of Devlin and Price (2007), Barense et al. (2009) investigated MTL activity during trial-unique novel face, object and scene oddity discrimination. The stimuli were presented from either identical or different viewpoints, a factor highly sensitive to MTL damage (King et al., 2004; Lee, Buckley, et al., 2005, 2006). Perirhinal activity was observed when participants distinguished between faces and objects presented from different, compared to identical, viewpoints (see Fig. 8c). By contrast, posterior hippocampus showed viewpoint effects for scenes, but also for faces. In a second study, Barense, Henson, Schwarzbauer, \& Graham (2008) compared activity in MTL regions for oddity judgements involving meaningful faces and objects (i.e., famous faces and everyday objects) and non-meaningful faces and objects (e.g., unfamiliar faces and nonsense objects such as "greebles"). Relative to a size oddity baseline, oddity judgements for all stimuli

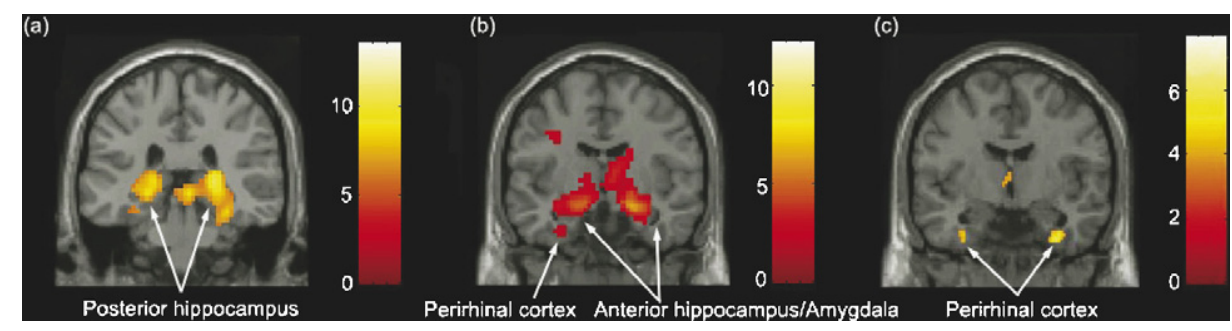

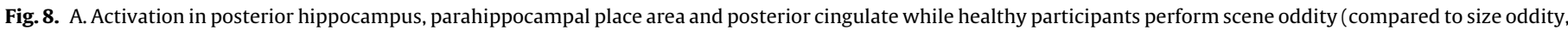

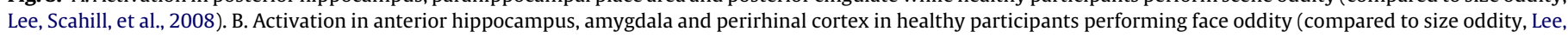
Scahill, et al., 2008). C. Activation in perirhinal cortex during object oddity, contrasted with size oddity (Barense et al., 2009). 
recruited regions within the MTL, including perirhinal cortex and hippocampus. Furthermore, familiarity effects were found in the hippocampus and perirhinal cortex (faces and objects) and temporal pole (faces only) bilaterally. This study confirms recruitment of MTL regions in healthy participants during oddity judgements for object and face stimuli, both when material is already familiar and when it is novel. Although these fMRI studies showed less convincing material-specific dissociations compared to the neuropsychological findings, they highlight that perirhinal cortex and posterior hippocampus show differential responses depending upon the type of stimuli to be discriminated.

As with Lee, Scahill, et al. (2008), one caveat regarding these fMRI studies is the difficulty of differentiating encoding-related activations from those associated with perception. It seems unlikely that the findings in Barense et al. (2009) can be fully explained by incidental encoding as there was no above baseline activity for same view conditions, which would have stressed encoding equivalently to the different view conditions. Addressing this issue more directly, O'Neil et al. (2009) found activity in right perirhinal cortex for face memory and oddity judgements for faces, and furthermore, activity in this structure distinguished between correct and incorrect trials in both conditions. This study confirms that an increase in declarative memory demands does not necessarily result in greater perirhinal cortex involvement, and highlights a growing consensus that this region may be more broadly involved in perception, as well as memory.

Of interest are some recent investigations that demonstrate similar distinctions between how the hippocampus and perirhinal cortex contribute to working and long-term memory for objects and scenes (Lee, Bandelow, Schwarzbauer, Henson, \& Graham, 2006; Litman, Awipi, \& Davachi, 2009; O'Neil et al., 2009; Pihlajamaki et al., 2004; although see Buffalo, Bellgowan, \& Martin, 2006; Diana, Yonelinas, \& Ranganath, 2008; Preston, Bornstein, Hutchinson, Gaare, Glover, \& Wagner, 2009). For example, Litman et al. asked participants to perform a 1-back task while viewing categories of stimuli (faces, objects, words, scenes and pseudowords); anterior perirhinal cortex and posterior parahippocampal cortex showed a dissociation, with the former more active for object stimuli and the latter for scene stimuli. While perirhinal cortex showed relatively selective activity for objects compared to other categories of stimuli, posterior parahippocampal cortex was also active for novel objects as well as for scenes. Further analysis revealed a graded decline in the response to scenes from posterior to anterior MTL cortical regions, but a flat and significant level of activity compared to baseline across all these structures for objects. Notably, the patterns of activations seen on simple object 1-back (Litman et al., 2009) and object oddity tasks (Barense et al., 2009; Devlin \& Price, 2007; Lee, Scahill, \& Graham, 2008; O'Neil et al., 2009) overlap with that observed when healthy participants are required to indicate object changes both within a trial (Lee, Bandelow, et al., 2006) and across trials (Pihlajamaki et al., 2004), as well as regions activated during fine-grained differentiation of objects (Bright, Moss, Stamatakis, \& Tyler, 2005; Moss, Rodd, Stamatakis, Bright, \& Tyler, 2005; Tyler et al., 2004) and cross-modal binding of object features (Taylor, Moss, Stamatakis, \& Tyler, 2006), confirming a key role for perirhinal cortex in object discrimination, and some degree of differential responsiveness to stimulus categories in MTL regions.

In summary, there is now a substantial body of fMRI research revealing complementary findings to those seen in amnesic patients. These two sets of data, when considered in conjunction with the animal studies which have the advantage of anatomical localisation at a level impossible in human neuropsychological investigations, reveal a number of intriguing findings challenging to accounts of human memory that propose a selective role for the MTL in long-term memory.

\section{Potential limitations of these findings}

The above review of the current literature highlights differential contributions from MTL structures, in particular the hippocampus and perirhinal cortex, to perception and memory for distinct categories of stimuli. These studies blur the boundary between perception and memory suggesting functional specialisation in the MTL along the lines of scenes and objects (including faces). In terms of object processing, convergent evidence (animal, neuropsychological and neuroimaging) now exists to support the view that perirhinal cortex is critical for discrimination of and memory for items with overlapping ambiguous features (see Bussey \& Saksida, 2007; Graham et al., 2007; Lee, Barense, et al., 2005; Lee, Buckley, et al., 2005; Lee, Bussey, et al., 2005). This evidence includes (a) virtually identical findings across species from the concurrent discrimination tasks of Bussey et al. (2002, 2003 see also Saksida et al., 2006), Barense et al. (2005 see Fig. 2) and Lee, Bussey, et al. (2005), (b) different patterns of performance on the high and low ambiguity trial-unique oddity object tasks designed by Barense et al. (2007) and (c) evidence of modulation of perirhinal cortex activity by the demand placed on integrating multiple object features (Barense et al., 2009; Devlin \& Price, 2007).

With regard to spatial stimuli, to date there has been no study that has systematically manipulated the spatial ambiguity between the simple geometric features of a scene and/or the spatial relationships between multiple objects within a scene in humans, so it remains unclear exactly what type of spatial ambiguity is particularly important. It is interesting to note, however, that monkeys with a fornix transection (a procedure which disrupts key input and output pathways to the hippocampus) show impairments when they are required to learn to discriminate 'tadpoles' on the basis of a conjunction of spatial features, such as the tadpole's position on the computer screen, tail length and tail angle. Deficits were particularly evident when the number of foils was increased or when there was spatial ambiguity between 'tadpoles', as measured by the size and length of the tail and the position on the computer screen). This finding is consistent with the possibility that the hippocampus is necessary for processing spatial conjunctions (Buckley, Charles, Browning, \& Gaffan, 2004).

A number of limitations and/or criticisms have been raised, however, regarding these findings, including (a) differences in experimental methodologies and stimuli across experiments (Baxter, 2009; Suzuki, 2009; Suzuki \& Baxter, 2009) and (b) differences in the localisation and/or extent of anatomical damage across patients (Baxter, 2009; Shrager et al., 2006; Suzuki, 2009; Suzuki \& Baxter, 2009). There have also been challenges to the explanations we have provided for our findings, such as the possibility that we have failed to address the importance of contributions from (c) long-term and/or (d) short-term memory (Levy et al., 2005; Shrager et al., 2006). We will discuss these issues in turn below, before outlining our theoretical model and concluding with a number of outstanding questions for future research.

\subsection{Differences in experimental methodology}

It is important to note that many of the published studies that test perception in amnesia often have significant methodological differences (even when 'similar' paradigms are used) and these inevitability limit attempts to make direct comparisons between experiments. For example, in a number of the experiments reported by Squire and colleagues, a task might be given 3 or 5 times, with averaged data from all presentations reported (Levy et al., 2005; Stark \& Squire, 2000), whereas in other studies deficits are typically evident after just one presentation of the task (Lee, Buckley, et al., 2005; Lee, Bussey et al., 2005; Lee, Buckley, et al., 2006, Experiment 2; Lee, Levi, Davies, Hodges, \& Graham, 2007; Barense et al., 2007). 
Paradoxically, perhaps, repetition of tasks might reduce any potential difference between controls and participants: Lee, Bussey, et al. (2005) report that repetition of morphed stimuli in their simultaneous visual discrimination task reduced performance in control participants (i.e., prior experience of stimuli resulted in an increasing number of errors during the task). If we had combined data from multiple presentations of our study, therefore, the difference between patients and controls present on the first experimental round would have been obscured.

A further key factor that may explain the lack of deficits in early studies of object and scene processing after MTL damage is the use of conditions/stimuli that do not maximise the possibility of observing an impairment. For example, Lee, Buckley, et al. (2005) found a deficit on oddity judgement for novel, but not familiar, objects; in Stark and Squire (2000) familiar and novel objects were included in the same condition, which may have reduced the possibility of observing an impairment in oddity judgement in their participants. While the subsequent findings from Barense et al. (2007) confirm that pre-experimental familiarity with stimuli does not preclude deficits on object discrimination in amnesic individuals, it is notable that this is typically for highly featural ambiguous stimuli and that, even then, our MTL patients consistently demonstrate better performance on familiar, compared to novel, stimuli (see Barense, Rogers, Bussey, Saksida, \& Graham, in press, for more details). In addition, and as discussed earlier in this article, the presence of unique and easily identifiable features in the blended scene stimuli used by Shrager et al.(2006), and the blended object stimuli used by Lee, Bussey, et al. (2005), may be the reason the patients show small, or no, impairment on these types of visual discrimination task.

It seems reasonable to propose, therefore, that the absence of deficits on object and spatial discrimination tasks may be due to a range of factors, including the use of stimuli which place an insufficient demand on feature ambiguity, differences in the size of the stimulus sets, and differences between paradigms (e.g., morphed versus odd-one out tasks). Furthermore, it is clearly better to adopt trial-unique paradigms, which provide a more stringent test of the perceptual hypothesis (e.g., Barense et al., 2007; Lee, Buckley, et al., 2005; Shrager et al., 2006). This proposal gains strength when one notes that, to date, proponents of the MTL 'memory' account have yet to demonstrate unequivocal evidence of intact perceptual processing on a task in which the degree of stimulus feature ambiguity is systematically controlled by explicitly manipulating the presence or absence of individual features (see Barense et al., 2007, for examples).

\subsection{Differences in location and extent of damage}

A clear limitation of our research has been the use of a ratingbased analysis of the degree of anatomical damage in our patients (Shrager et al., 2006; Squire et al., 2006; Suzuki, 2009). This measure confirmed that the hippocampal group had significant atrophy of the anterior hippocampus, but were not different from controls in any other brain region (anterior temporal lobe, amygdala, lateral temporal lobe, posterior hippocampus, parahippocampal gyrus (corresponding to entorhinal cortex), medial bank of the collateral sulcus (corresponding to transentorhinal cortex), lateral bank of the collateral sulcus (corresponding to perirhinal cortex) and medial bank of the occipitotemporal sulcus). The MTL group showed atrophy in all regions, other than the lateral temporal lobe measure (see Lee, Bussey, et al., 2005, for details).

It has been proposed that the hippocampal cases reported by our group may have additional parahippocampal involvement that caused perceptual difficulties with scene discrimination (Squire et al., 2006). Similarly, Hartley et al. (2007) discussed this possibility as a potential explanation for the variability in their hippocampal cases on tests of scene perception versus scene memory. For example, Hartley et al. (2007) proposed that the hippocampus forms a viewpoint independent schema of the layout of an environment, a representation that is particularly critical for memory processing (e.g., when a delay is introduced, see Bird \& Burgess, 2008a for a more detailed discussion). By contrast, posterior parahippocampal cortex may store a viewpoint-specific spatial representation of the environment that is less adaptable (Epstein, 2008; Epstein, Graham, \& Downing, 2003; although see Bar, Aminoff, \& Schacter, 2008; Diana et al., 2008; Ewbank, Schluppeck, \& Andrews, 2005). While this viewpoint-specific representation may enable one to solve a scene discrimination task, it is not sufficiently flexible to support memory for scenes. A key prediction from this view is that damage to the hippocampus will result in spatial memory impairments only. When damage extends beyond the hippocampus into posterior parahippocampal cortex, however, spatial perceptual deficits will also be seen.

Until recently it has been difficult to discount the potential contribution of parahippocampal damage in our patients. In the original rating analysis, two of three hippocampal cases showed minor involvement of the crown of the parahippocampal gyrus (scoring 0.75 out of 4 , with a larger score reflecting more damage). Notably, however, this rating was of a region corresponding to entorhinal cortex, rather than posterior parahippocampal cortex, and consequently it was not clear whether posterior regions were implicated in these cases. This question has now been addressed by recent volumetric studies demonstrating that our hippocampal cohort show selective damage to the hippocampus in the context of no significant obvious atrophy to posterior parahippocampal cortex (see Lee, Gough, Davies, Barense, \& Graham, 2008). While these types of analyses are not definitive, they confirm our assertion that the hippocampus, perhaps in conjunction with posterior parahippocampal cortex, is necessary for spatial discriminations as well as spatial memory.

Similar arguments about potential additional structural damage beyond the perirhinal cortex have been made about the MTL group we have tested: for example, it has been proposed that the perceptual deficits in object and face processing seen in cases with more extensive MTL lesions may reflect broader damage beyond perirhinal cortex, in particular to lateral temporal lobe regions, such as fusiform gyrus (Shrager et al., 2006). While it is true that one of our patients does have a lesion that extends-based on both rating and volumetric analysis-to more lateral cortex and involves the middle and superior temporal gyri, it is notable this is not true of all our cases who show remarkably similar patterns of performance across the tests, consistent with their overlapping damage to perirhinal cortex (Lee, Gough, et al., 2008). In addition, none of our patients have shown deficits with simple object perception, such as when a single feature is sufficient to support identification of objects, even during rapidly learnt discriminations (Barense et al., 2005), and their performance on (a) very difficult colour, size and shape oddity tasks and (b) simple object (low ambiguity and same view) oddity judgements is typically good (Barense et al., 2007; Lee, Buckley, et al., 2005). This pattern is consistent with that seen in non-human primates: fine-grained colour discrimination is impaired in macaque monkeys after damage to lateral temporal areas (area TE), but not after selective perirhinal lesions (Buckley et al., 1997; Bussey et al., 2002; Lehky \& Tanaka, 2007), and monkeys with perirhinal lesions show normal performance on same view oddity tasks (Buckley et al., 2001).

Considering the impact of extent of lesion, it is important to note that our findings in amnesic individuals-and those of other researchers-have typically been based on contrasting a group of patients with hippocampal damage with a group of cases with larger lesions involving both the hippocampus and other MTL structures. A downside of this 'subtractive' approach is the possibility 
that the differential profiles seen between the HC and MTL patients may be due to inevitable differences in the degree of hippocampal involvement or overall lesion size in the two groups, rather than the additional involvement of perirhinal cortex in the MTL participants. For example, if memory for scenes was inherently more difficult than memory for objects and/or faces (as seen in Taylor, Henson, \& Graham, 2007), perhaps because humans have particular expertise in face and/or object identification, then it might be possible to explain differences in memory and/or discrimination for distinct types of stimuli as an effect of stimulus difficulty interacting with differences in the volume of MTL damage present across patient groups. More specifically, individuals with smaller MTL lesions, such as those with hippocampal damage, might show impaired performance on scenes but not faces because the former are more difficult than the latter. By contrast, individuals with broader MTL lesions may show impairments for both categories of stimuli due to more extensive damage to this 'memory' system. Consequently, the profiles demonstrated by our amnesic patients could indicate category-specific roles for scene and object processing within different MTL structures or they could just reflect an interaction between the overall amount of MTL damage and differences in difficulty across task conditions.

A key prediction of the former, but not the latter, account is that it should be possible to see the opposite pattern of performance (e.g., impaired face discrimination in the context of good scene discrimination when there is perirhinal involvement that spares the hippocampus). This pattern has been reported. Lee, Buckley, et al. (2006) found that patients with Alzheimer's disease, which affects functioning of the hippocampus, parahippocampal cortex and retrosplenial cortex early in the disease with less involvement of perirhinal cortex (Davies, Graham, Xuereb, Williams, \& Hodges, 2004; Nestor, Fryer, \& Hodges, 2006; Nestor, Fryer, Ikeda, \& Hodges, 2003a; Nestor, Fryer, Smielewski, \& Hodges, 2003), showed poor scene discrimination in the context of good face oddity judgement. By contrast, individuals with semantic dementia, in whom there is early involvement of perirhinal cortex and anterior hippocampus (Davies et al., 2004), but with preservation of other diencephalic regions thought to be important for memory (Nestor et al., 2006), showed the opposite profile of impairment (normal scene oddity judgement but poor face and object processing). This pattern has also been replicated in other paradigms (Barense et al., in press; Lee et al., 2007; see Graham, Lee, \& Barense, 2008, for review) as well as in tests of recognition memory for faces and scenes (Cipolotti \& Maguire, 2003) and clearly indicates that differences in difficulty between classes of stimuli are unlikely to explain the functional patterns exhibited by our patient groups.

\subsection{Failure to benefit from long-term memory?}

Some researchers have proposed that when perceptual impairments are seen in amnesia they may reflect a failure to benefit from long-term memories that contribute to online object and scene processing (Levy et al., 2005; Ryan \& Cohen, 2004; Shrager et al., 2006). According to this view, the MTL plays a specialised role in long-term declarative memory, consistent with existing theoretical accounts of memory, and the perceptual deficits observed in amnesic individuals reflect an inability to access MTL-dependent declarative memories that healthy participants can use to support performance on perceptual discrimination tasks.

There is increasing empirical support that the perception of scenes, and presumably objects, is an interactive process influenced by prior episodic experience with similar stimuli and conceptual knowledge about item and scene categories (Henderson \& Hollingworth, 1999; Henderson, Weeks, \& Hollingworth, 1999; Hollingworth, 2004, Hollingworth, 2005, Hollingworth, 2007; Hollingworth, Williams, \& Henderson, 2001; Summerfield, Lepsien,
Gitelman, Mesulam, \& Nobre, 2006). For example, Henderson (2003) reviewed evidence that the control of gaze during scene processing is influenced by prior exposure to similar scenes. This includes episodic information that is gathered during exposure to a single scene (short-term) and over many encounters with the same or similar scenes (long-term). For example, participants will fixate a point in a scene that previously contained a relevant object (Hollingworth \& Henderson, 2002). Furthermore, previous experience with a scene will cue participants to the location of a search target within a scene, including one that is indiscernible from peripheral vision (e.g., small T/L's superimposed onto scenes, Brockmole \& Henderson, 2006; Chun \& Jiang, 1998), and improves reaction times for object detection, as well as accuracy (Summerfield et al., 2006). Similarly, semantic and/or spatial knowledge about the environment and the objects in it influences how we attend to presented scenes, including guiding the direction of the first saccade made during scene presentations, such as searching for an object in an appropriate location (Henderson et al., 1999). For example, if participants are required to search for people in a scene, they will rarely make their first saccade upwards as people are unlikely to be found in the sky (Torralba, Oliva, Castelhano, \& Henderson, 2006).

MTL structures may be responsible for some of these effects. Using a paradigm in which participants were asked to detect a small key in previously familiar versus novel scenes using either memory or a visual cue, Summerfield et al. (2006) found a network of brain regions common to both memory-guided and visuallyguided orienting (e.g., intraparietal sulcus, angular gyrus, anterior and posterior cingulate, fusiform gyrus, posterior hippocampus and parahippocampal gyrus), but additionally revealed activity in the hippocampus during memory-guided orientation, and a significant relationship between hippocampal activity and the behavioural advantage conferred by prior experience. It seems clear, therefore, that the way in which we perceive is rapidly and flexibly influenced by our ongoing episodic experience, and that this involves interactions between MTL structures and perceptual representations. Furthermore, an inability to form and utilise this episodic information is likely to have a significant impact on the ability to perceive (e.g., a deficit in perception may arise from a difficulty with memory).

There are a number of reasons why we believe this view cannot fully explain our data. First, the beneficial effects of prior exposure on scene processing, and presumably on other types of stimuli, are often seen on the first fixation and saccade (e.g., on the direction of our early fixations to relevant locations and/or objects). While amnesic individuals may well not demonstrate these early advantages compared to controls, it is not immediately clear how this could account for impairments on tasks in which the participants have as long as possible to make their decision (Lee, Buckley, et al., 2005). Second, when there is evidence of long-term memory contribution over time, such as improved accuracy and/or reaction time (Summerfield et al., 2006), this is typically in situations in which stimuli are repeated. As the deficits seen in our patients are significant even in the initial test block prior to the repetition of stimuli (Experiment 1, Lee, Buckley, et al., 2005), and on completely novel trial-unique items (Barense et al., 2007; Experiment 2, Lee, Buckley, et al., 2005; Lee, Buckley, Pegman, Spiers, Scahill, Gaffan et al., 2006), it seems unlikely that this is the full explanation for the findings. Third, as we observe impairments on tasks that have utilised familiar, as well as novel, scenes, objects and faces, it seems unlikely that difficulties in making use of generic semantic and spatial knowledge about scenes and objects can underlie these deficits. In fact, we know that patients with MTL lesions show better, albeit impaired, performance on familiar compared to novel items, a finding that is in keeping with a beneficial contribution of semantic knowledge on perceptual discrimination, but 
not with an account in which lack of access to this information is the underlying cause of the problem (Barense et al., in press). In summary, therefore, we are supportive of an account in which perception is influenced and facilitated by our prior experience and semantic knowledge, and agree that there are behavioural and neural consequences of these interactions (see Cumming, Patterson, Verfaellie, \& Graham, 2006; Graham, Simons, Pratt, Patterson, \& Hodges, 2000; Graham, Patterson, Powis, Drake, \& Hodges, 2002; Majerus, Norris, \& Patterson, 2007; Patterson, 2007; Patterson et al., 2006; Patterson, Nestor, \& Rogers, 2007; Rogers, Patterson, \& Graham, 2007). Whether this can fully account for the patterns seen in our patients seems unlikely, given that these individuals show deficits in scene and object discriminations for novel trial-unique stimuli, even when there is unlimited time to make the decision.

\subsection{Failure to utilise short-term memory?}

In a similar vein, it has also been proposed that our findings may reflect an impairment in very short-term memory, such as - in the case of the oddity judgement tasks - the inability to hold information online across the saccades required to compare simultaneously presented stimuli (Ranganath \& Blumenfeld, 2005; Ranganath \& D'Esposito, 2001). While this proposal probably provides the strongest challenge to EMA, there are still outstanding issues regarding how this view fully explains our findings. In a review on the role of the MTL in working memory, Ranganath and Blumenfeld (2005) suggest that there may be more evidence of deficits in working memory in amnesia than previously believed (see Fig. 9a, Aggleton, Shaw, \& Gaffan, 1992; Buffalo, Reber, \& Squire, 1998; Holdstock et al., 1995; Holdstock, Gutnikov, et al., 2000; Owen, Sahakian, Semple, Polkey, \& Robbins, 1995; Ryan et al., 2000; Ryan \& Cohen, 2004). This view has gained increasing support from a series of recent neuropsychological investigations (Hannula, Tranel, \& Cohen, 2006; Hartley et al., 2007; Olson, Moore, Stark, \& Chatterjee, 2006; Olson, Page, Moore, Chatterjee, \& Verfaellie, 2006), in which amnesic individuals demonstrated impaired working memory, particularly when conjunctions of stimuli (object-place, Olson, Page, et al., 2006) or relations amongst elements within a scene (faces-scenes, Hannula et al., 2006; computer-generated mountains, Hartley et al., 2007) were tested. For example, Hannula et al. (2006), tested participants with amnesia secondary to an anoxic episode on recognition memory for relations among items embedded within scenes and for face-scene pairs. The patients showed poor memory in both conditions, even at the shortest lag when the studied item immediately preceded the test probe (see Fig. 9b and c). Similarly, in Olson, Page, Moore, Chatterjee, \& Verfaellie (2006) item memory for individual objects and locations was preserved, but memory for object-place conjunctions was impaired (although see Shrager, Levy, Hopkins, \& Squire, 2008). Furthermore, as discussed earlier in this article, Hartley et al. (2007), found that all four of their hippocampal cases were impaired at scene working memory after a two second delay.

Consistent with the patient data, fMRI studies have revealed activation in MTL regions during short-term memory tasks. For example, Ranganath and D'Esposito (2001) found bilateral anterior hippocampal activation during active maintenance of faces
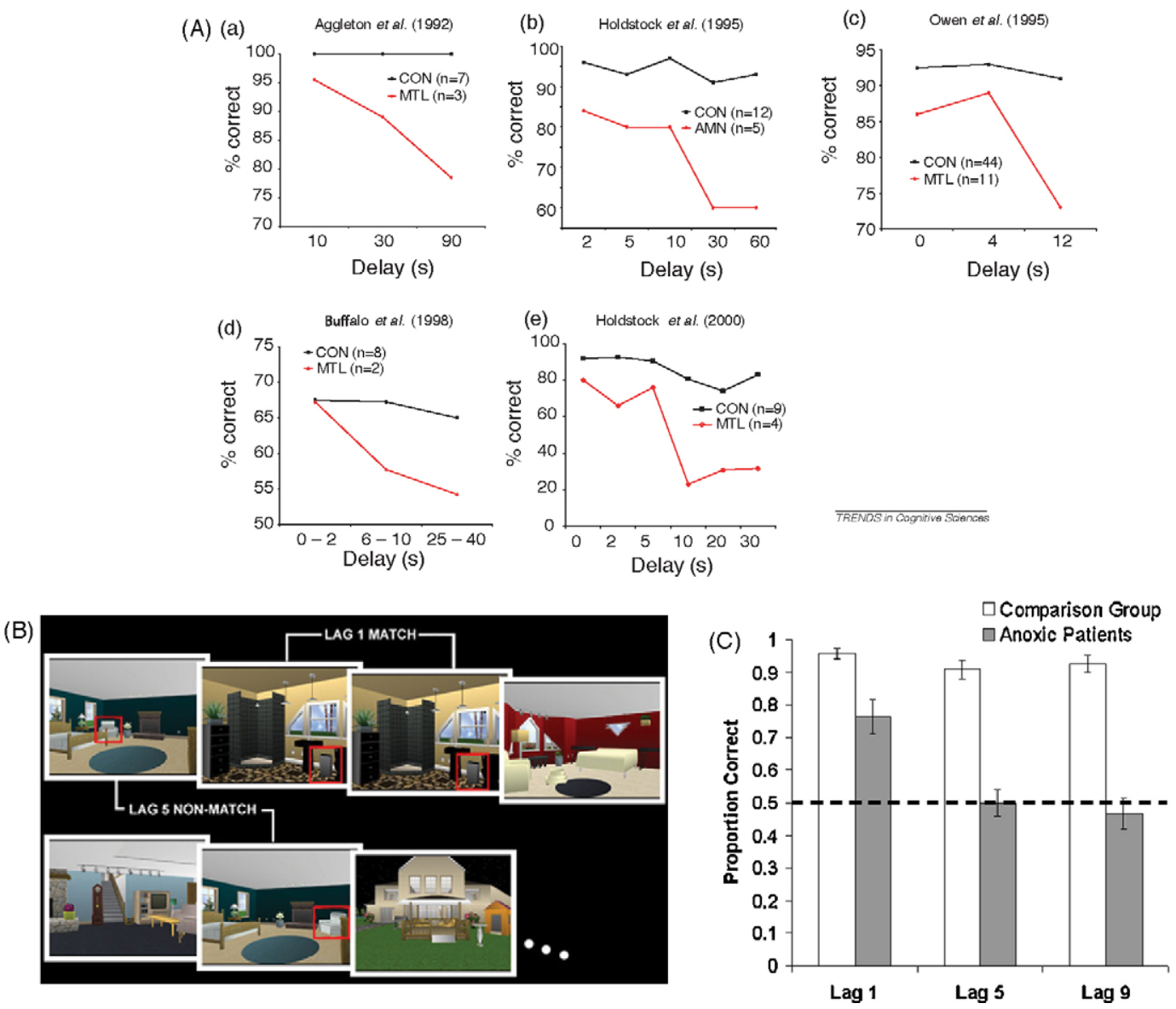

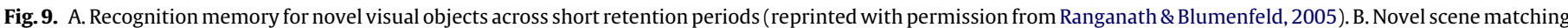

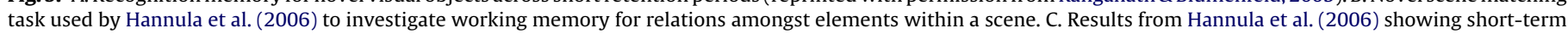

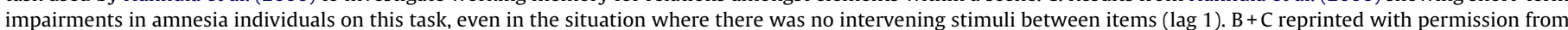
Hannula et al. (2006). 

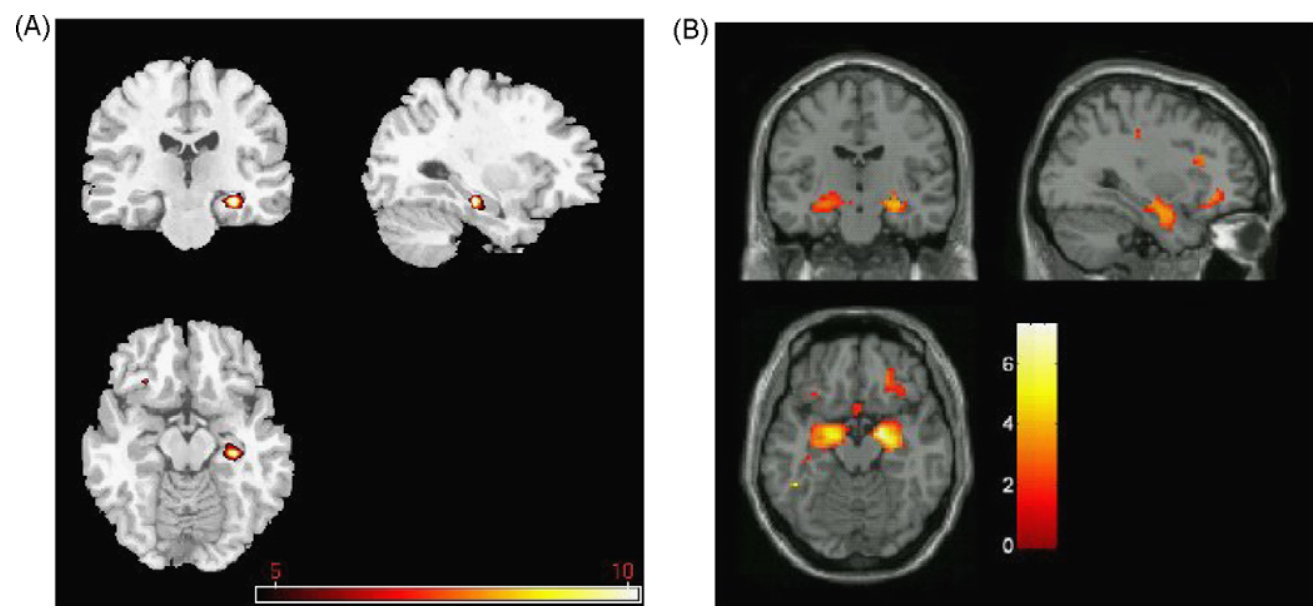

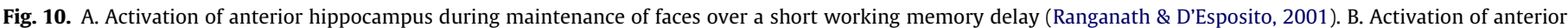
hippocampus during oddity judgement for faces (Lee, Scahill, et al., 2008).

over a short delay, but not during face encoding or recognition (see Fig. 10a). Interestingly, this activation is virtually identical to Lee, Scahill, et al.'s (2008) anterior hippocampal activation during face oddity judgements (see Fig. 10b). More recently, Hannula and Ranganath (2008) asked participants to mentally rotate a grid containing four objects and then indicate whether the relative location of the objects matched a test stimulus. Although the delay between study and test was only $11 \mathrm{~s}$, comparison of correct versus incorrect trials revealed greater activity at encoding in the left anterior and posterior hippocampal regions, as well as in perirhinal cortex bilaterally. Activity in the hippocampus was greater for 'match' compared to 'mismatch' trials, a finding consistent with a role for this region in processing short-term relational or binding information about the particular locations of the objects.

These studies clearly demonstrate a role for the hippocampus in short-term memory, particularly when relational processing is required. Impairment in short-term memory is consistent with our view, which predicts deficits in both short-term and long-term memory, regardless of delay, as long as the task taxes representations dependent upon the perirhinal cortex and/or hippocampus. Notably, however, it is controversial as to whether the hippocampus is necessary for all types of relational processing (Aggleton et al., 2007; Barense et al., 2007; Hartley et al., 2007; Konkel, Warren, Duff, Tranel, \& Cohen, 2008; Kumaran \& Maguire, 2005). For example, our hippocampal cases showed normal object processing even when they were required to discriminate between conjunctions/relations of features within individual objects (Barense et al., $2005,2007)$. This finding highlights the involvement of the hippocampus to short-term relational processing of items that are perceptually distinct rather than binding of features within individual objects (see Eichenbaum, 2004, for further discussion of this issue). Furthermore, it is notable that short-term working memory or perceptual impairments are most commonly elicited in amnesic individuals with hippocampal damage when the task places a demand on spatial processing (Hartley et al., 2007; Shrager, Levy,et al., 2008; Shrager, Kirwan, \& Squire, 2008; although see Konkel et al., 2008). For example, in Hartley et al. (2007), patients with hippocampal damage showed normal performance on a nonspatial task in which they were required to process conjunctions of multiple environmental features that were not spatially related (lighting, cloud cover and colour of vegetation). Similarly, Kumaran and Maguire (2005) found that navigation within a spatial, but not social, network activated the hippocampus, despite both conditions placing a demand on processing 'relations'. The hippocampus may not be necessary, therefore, for some types of relational processing (see also Aggleton et al., 2007), but does appear to be essential for relational processing of objects embedded in a scene (Hannula \& Ranganath, 2008; Hannula et al., 2006) or between spatial features in the environment, even over extremely short delays (Hartley et al., 2007).

While our account readily explains short-term memory deficits-damage to representations in the MTL will lead to deficits on memory tasks that require these representations-it remains a challenge for proponents of a short-term memory view of MTL function to explain (a) why these effects should be distinguished by stimulus-category (e.g., why hippocampal damage would lead to a short-term memory impairment for scene stimuli, but not for objects and faces) and (b) to defend the necessity of proposing a selective mnemonic role for MTL-at the level of trans-saccadic memory (e.g., 100-200 ms). Indeed, a recent fMRI study found that greater working memory demand-as measured by a 2-back condition, in which participants detected stimulus repetitions separated by a single image, versus a 1-back condition, in which participants detected two identical images presented successively-was associated with increased posterior hippocampal and parahippocampal cortex activity when complex virtual reality rooms were presented, but not when 2D spatial arrays were shown (Lee \& Rudebeck, 2009). This finding supports the idea that the MTL does not play a general role in short-term working memory and that stimulus type is a critical determinant of MTL involvement in both perceptual and mnemonic tasks.

\section{Outstanding questions and future directions}

In summary, while there are criticisms that can be levelled at the experiments discussed in this review, and discrepancies between studies that need to be addressed, there is now significant evidence of a functional specialisation in how MTL structures contribute to memory and perception of objects and scenes, and a growing sense of agreement that traditional fractionations of memory (such as between short-term and long-term memory) may not be the best way to explain existing neuropsychological and neuroimaging memory data. For example, in the context of discussing the working memory deficits seen in Buffalo et al. (1998) and Ryan and Cohen (2004), Olson, Page, et al. (2006) note: "When hippocampally based WM (working memory) deficits have been observed in the past, the data collide with the paradigmatic view of memory and the hippocampus: that it is only important for LTM (long-term memory). ... These results [the authors are referring to Buffalo et al. (1998) and Ryan and Cohen (2004)] were interpreted as being attributable to the influence of impaired long-term memory in amnesia. Unfortunately, the circular logic of this interpretation (e.g., if amnesia is 
defined as an impairment in long-term memory then any memory impairment, regardless of delay, must be an impairment in long-term memory) renders it impotent. (p4599)".

We have put forward a more controversial extension of this view here (EMA) in which there is a blurring of the boundary between perception and memory, with memory dependent upon the activation of distributed representations stored in the visual ventral stream, including complex conjunctive object and scene representations stored in the perirhinal cortex and hippocampus, respectively. The key features of EMA are (a) that the role of the MTL is not restricted to long-term memory, but extends to short-term memory, implicit memory and perception; (b) that regions beyond the MTL, such as extrastriate cortex, are also capable of supporting memory (Cansino, Maquet, Dolan, \& Rugg, 2002; Harrison \& Tong, 2009; Lopez-Aranda et al., 2009; Prince, Dennis, \& Cabeza, 2009); (c) that the role played by different MTL regions in memory and perception can be dissociated by type of visual material; and (d) that MTL regions are involved in memory and perceptual tasks only when these place a demand on processing complex conjunctive representations. This view rejects the idea of a specialised MTL memory system (see also Bussey \& Saksida, 2007; Gaffan, 2002; Horel, 1978). For example, paraphrasing Olson, Page, et al. (2006), when perceptual deficits have been observed in amnesia, the data collide with which ever view of memory (i.e., a role in either long-term or short-term memory) and the hippocampus the individual believes, and the impairment must therefore be a consequence of this failure of memory. Instead, a more parsimonious explanation for the findings described here is that memory emerges from hierarchically organised perceptual representations distributed throughout the brain (see also Buckley \& Gaffan, 2006; Bussey \& Saksida, 2005) in conjunction with top-down controlled processes based on motivational goals and task demands (Duncan, 2001). This view is consistent with animal studies in which dense amnesia is not caused by removal of specific cortical sites, such as MTL structures, but is seen when there is widespread disconnection of temporal cortex, basal forebrain and midbrain connections (Easton \& Gaffan, 2000, Easton \& Gaffan, 2001; Easton, Parker, \& Gaffan, 2001; Gaffan, Parker, \& Easton, 2001; Maclean, Gaffan, Baker, \& Ridley, 2001).

While this review has revealed a number of confirmatory findings supportive of EMA, it has also raised a number of outstanding questions, such as (a) how would activations in the MTL be modulated by process (e.g., by recollection and familiarity for different stimulus types) and (b) how does our model explain other key phenomenon of the amnesic syndrome, in particular delay-dependent effects and deficits in verbal memory?

\subsection{Representations or process?}

As discussed in the Introduction, one of the most influential models of the MTL proposes that the hippocampus and perirhinal cortex differentially contribute to recollection and familiarity (Brown \& Aggleton, 2001). This view does not make any predictions about how these processes may be influenced by stimulus. One explanation is that scenes place more demand upon the processes presumed to be dependent upon the hippocampus, such as recollection or processing of relations across the different elements comprising a scene. By contrast, processing faces or objects holistically as a gestalt (Tanaka \& Farah, 1991; Young, Hellawell, \& Hay, 1987) may increase reliance upon a signal detection-like familiarity process dependent upon perirhinal cortex (Brown \& Aggleton, 2001; Haskins, Yonelinas, Quamme, \& Ranganath, 2008; Yonelinas, Kroll, Dobbins, \& Soltani, 1999). This explanation can account for amnesic cases who show deficits in scene, but not face, recognition memory after selective hippocampal involvement (Bird et al., 2008) and for participants who demonstrate impairments in recognition memory for both faces and scenes after damage to both the hippocampus and perirhinal cortex (Cipolotti et al., 2006; Taylor et al., 2007).

As discussed by Taylor et al. (2007, see also Bird \& Burgess, 2008; Bird et al., 2008), however, it is not immediately clear that a simple distinction between recollection and familiarity is sufficient to explain these stimuli-specific effects. For example, intact recollection of faces (as measured using ROC analyses) has been reported after focal hippocampal damage (e.g., VC, Cipolotti et al., 2006; Jon et al., 2008; RH, Bird, Shallice, \& Cipolotti, 2007), a pattern that implies that recollection may not be solely mediated by hippocampal structures (see also Awipi \& Davachi, 2008; Diana et al., 2008; Staresina \& Davachi, 2008). Similarly, Awipi and Davachi (2008) investigated the contribution of MTL regions to recollection and familiarity for scenes with novel repeating objects. Participants performed a scene-object encoding task in the scanner, and subsequently indicated whether a scene had been previously viewed and which object (out of six) had been paired with it. Activation in the right perirhinal cortex was greater for trials in which both scene and object were correctly recognised (source memory) compared to trials in which the scene (but not object) was correctly remembered and also trials in which neither the scene nor object were recognised (misses). This finding was also evident when the trials were further split according to the participants' remember/know decisions: greater activity was seen in perirhinal cortex for scene + object 'remember' trials when contrasted with correct scene 'remember' trials. Parahippocampal cortex activity, by contrast, was greater for the scene 'remember' trials only (see also Preston et al., 2009, for evidence of a linear trend in subsequent memory activity in scene-specific voxels in parahippocampal cortex). Similarly, posterior hippocampus showed greater activity for 'remember' trials in which just the scene was recognised compared to trials in which both scene and object were correctly recognised, a pattern that was dissociable from an anterior hippocampal region that showed a content-independent profile of response (e.g., similar activation for both these response categories).

These studies highlight the possibility-that we would also currently favour-that activity in MTL regions does not clearly fractionate along the lines of recollective- and familiarity-based processes alone (see also Squire et al., 2007), and consequently that memory process cannot explain the differential effects of stimulus category observed in fMRI studies (Awipi \& Davachi, 2008; Barense et al., 2009; Lee et al., 2007; Lee, Scahill, et al., 2008; Litman et al., 2009), and in our patients (Lee, Buckley, et al., 2005; Lee, Bussey, et al., 2005; Taylor et al., 2007). Instead, we propose that the creation of object versus scene representations reflects a key division in how the MTL is organised, with the caveat that any differential sensitivity in the processing of different types of stimuli may not be total (e.g., posterior hippocampus was also activated for faces in Barense et al. (2009) and for objects in Litman et al. (2009)). Our view predicts that representations in the perirhinal cortex and posterior hippocampus are capable of supporting both recollection and familiarity depending upon the demands of the task (e.g., the requirement for processing of object and scene information), although unlike Squire et al. (2006), in our account, recollection and familiarity effects in each MTL region will be modulated by the type of visual stimuli supported by that structure. It is important to note that this hypothesis has not yet been completely tested: while there is some evidence that perirhinal cortex can support recollection as well as familiarity for objects (Awipi \& Davachi, 2008; Duarte, Henson \& Graham, submitted for publication; Staresina \& Davachi, 2008), there is less support for the view that familiarity effects can be seen in the hippocampus (although see Gold et al., 2006; Kirwan et al., 2008; Shrager, Kirwan, et al., 2008), or that these familiarity effects are modulated by stimulus type. For example, Montaldi, Spencer, Roberts, \& Mayes (2006) investigated scene 
memory at retrieval, and found decreasing activity in perirhinal cortex as participants' perceived familiarity for scenes presented previously increased. The hippocampus showed no modulation of activity by familiarity levels, but instead was significantly active for scenes judged as 'recollected' compared to those that possessed strong familiarity. This finding is a challenge to EMA as we would predict that both recollection and familiarity would be driven by scene representations located in the hippocampus. It remains possible, however, that this perirhinal familiarity effect was driven by memory for objects and items present in the scene, rather than the scenes themselves and/or, as argued by Squire et al. (2007), that the lack of hippocampal activation for familiarity reflects a failure to detect weaker memories (memory strength account). Although our account has some similarity to the memory strength view, in that we believe that the hippocampus and perirhinal cortex can support recollection and familiarity, it differs by remaining faithful to the idea that there are functional differences, in our view relating to material type, in how MTL regions contribute to perception, and consequently to memory.

EMA shares some features with a recently proposed theory, the Binding of Item and Context model (BIC, Diana et al., 2007, 2009; Eichenbaum et al., 2007). Like EMA, this theory proposes that the type of information processed by MTL regions is more important than a simple division between recollection and familiarity. For example, as noted earlier in the article, BIC proposes that the perirhinal cortex processes item information and supports familiarity, while recollection is dependent upon the hippocampus and parahippocampus. The former binds items with context, while parahippocampal cortex stores any type of contextual information considered separate from the target at encoding. BIC proposes, therefore, that the way in which a participant encodes information, for example either as an item or as context, modulates involvement of MTL structures in long-term memory (see Diana et al., 2009).

There are some key differences between BIC and EMA. First, while BIC assumes that perirhinal cortex supports familiarity for all material types when these are processed as items, EMA predicts that perirhinal cortex supports familiarity for some types of material (e.g., objects and faces), but not others, with posterior hippocampus playing a key role in familiarity, and recollection, for scenes. Related to this, a second distinction is how these views explain the role of posterior hippocampus and parahippocampal cortex in scene memory (Lee, Scahill, et al., 2008; Taylor et al., 2007). While we have proposed that these regions encode and store complex conjunctive scene representations that support both perception and memory (Barense et al., 2009; Graham et al., 2006; Lee, Buckley, et al., 2006), BIC predicts that these patterns reflect the requirement to bind objects within a scene, effects which would not necessarily be restricted to scene stimuli but could be extended to any situation in which there was a requirement to bind an item with a context (spatial and non-spatial, e.g., face-colour). This proposal has some similarities to the domain-dichotomy view in which the hippocampus is necessary for cross-, but not within-, modality associations (Mayes et al., 2007). Unlike BIC, EMA also predicts that MTL regions are only necessary for memory when there is a requirement to process representations with complex overlapping features (Barense et al., 2005). By contrast, memory for more simple items (e.g., shapes, colour, and so on) may be dependent upon other regions in the visual ventral stream, and not MTL (see Harrison \& Tong, 2009; Lopez-Aranda et al., 2009). As preliminary support for this prediction, Mundy, Downing, Dwyer, Honey, \& Graham (submitted for publication) found that activity in perirhinal cortex and posterior hippocampus, but not posterior fusiform gyrus and parahippocampal gyrus, was modulated by accuracy (correct > incorrect) in discrimination learning of pairs of conjunctive faces and scenes, respectively (see Graham et al., 2006, for complementary data from patients with MTL involvement). Dis- crimination learning of checkerboards (Mundy et al., 2009) and dots (Mundy et al., submitted for publication), activated visual cortex but not extrastriate or MTL regions. A final important difference between BIC and EMA is that the latter extends the role of the MTL beyond mnemonic processing to include complex perceptual discrimination, whereas BIC focuses on a purely mnemonic role for the MTL and has yet to address why MTL regions may play a role in trialunique perceptual discriminations that place minimal demand on long-term memory.

\subsection{Delay-dependent effects and deficits in verbal memory}

A challenge for the future is to understand how our model might account for the full phenomenology of the amnesic syndrome, as well as the various nuances of normal memory. At first glance, it may seem that EMA is rather limited in its ability to explain the other deficits in amnesia (e.g., delay-dependent memory effects and impairments in amnesic individuals that are not overtly spatial, such as difficulties with word learning and poor recall of a prose passage), but we believe there are potential explanations that address some of these issues, and clear experiments that would test our account directly.

Addressing delay-dependent deficits, as EMA predicts that perception and memory should always go hand-in-hand (i.e., the memory impairment arises due to difficulties with the initial representation), the answer to this question initially seems simple-delay is just one means of making a task more difficult. There are at least two, potentially related, factors that may contribute to this: (a) decay and (b) interference. More specifically, the longer the delay between processing a representation and using information about the item at test, the harder it will be to activate this representation due to decay over time. This problem will be exacerbated when a representation is partially damaged, which may accelerate decay. Thus, an amnesic individual may show little evidence of a deficit compared to controls at simultaneous time-points (when discrimination may be close to ceiling) but impairment when tested at longer delays. Furthermore, as suggested by Bussey and Saksida (2007) with respect to perirhinal cortex, a delay between encoding and test is likely to result in interference from encountering aspects of the same simple features that make up the study item (e.g., edges, colours and so on) in other stimuli presented during the delay. The presence of these overlapping features in other items will result in feature ambiguity-increased familiarity with these simple features-and the problem will no longer be solvable using a single feature strategy. A direct prediction from this explanation is that the larger the delay, the greater the likelihood of intervening ambiguous features and consequently, the larger the recognition memory deficit (see Cowell et al., 2006, for a connectionist model of perirhinal cortex that demonstrates this pattern).

The key point here is that, although impairment is particularly evident after a delay, this does not necessarily imply that the deficit is in memory per se: due to impoverished representations of complex feature conjunctions, amnesic individuals with perirhinal damage are forced to rely on undamaged simple featural representations. These simple featural representations may not be sufficient to solve the task, especially when there is a delay between study and test. One prediction from this account is that object recognition memory-at all delays-should be preserved after selective hippocampal damage (see Holdstock, Gutnikov, et al., 2000; Holdstock, Mayes, Cezayirli, Isaac, Aggleton, et al., 2000; Mayes et al., 2002; Vargha-Khadem et al., 1997, for supportive evidence, although see also Gold et al., 2006; Hamann \& Squire, 1997; Manns et al., 2003; Reed \& Squire, 1997; Wais et al., 2006 for counter examples). A second prediction is that damage to the perirhinal cortex should affect memory for high ambiguity object stimuli (at all time-periods) but not low ambiguity object stimuli. This hypothesis 
has yet to be tested systematically in individuals with MTL damage, but consistent with this prediction, rats with perirhinal and postrhinal lesions have been shown to be impaired at high, but not low, ambiguity discriminations on zero-delay spontaneous object recognition (Bartko et al., 2007a).

A further challenge to our account is why word learning and memory for a prose passage is so sensitive to MTL damage. Although, given the nature of the tests that we have utilised, our view has focused on spatial processing, it is clear that the hippocampus is also important for aspects of temporal, as well as spatial, processing (see Bird \& Burgess, 2008a; Kumaran \& Maguire, 2009 , for further discussion of this issue). Thus, it is perhaps no surprise that retrieval of information spaced over time (e.g., word lists or prose passages) is likely to be highly sensitive to hippocampal damage. What is not clear, however, is how these two types of context interact or whether they have different neural substrates. There is empirical evidence that our understanding of time, and consequently the way in which we use it is dependent upon our physical experience and representations of space (Alverson, 1994; Boroditsky, 2000; Boroditsky, 2001; Bowerman, 1983; Casasanto \& Boroditsky, 2008). Consistent with a strong psychological relationship between these two constructs, electrophysiological studies have demonstrated that hippocampal place cells, which fire when a rat is in a particular location within an environment (O'Keefe, 1976, O’Keefe, 1991; O’Keefe \& Burgess, 1996; O’Keefe, Burgess, Donnett, Jeffery, \& Maguire, 1998; see Ekstrom et al., 2003, for data in humans), can also encode temporal sequence information (Buzsaki, 2002; Dragoi \& Buzsaki, 2006; Manns, Howard, \& Eichenbaum, 2007).

Extending these findings to humans, Kumaran and Maguire (2006) used a repetition fMRI procedure to investigate the contribution of MTL regions to memory for temporal events. Participants were shown sequences of four objects that were re-presented after a short $3 \mathrm{~s}$ delay either in the same order as seen previously or in a recombined order and were asked to indicate when the same object was seen twice in a row. Comparison of activity for a reordered sequence of objects compared to the same sequence of objects revealed increased activity in the left hippocampus and the right entorhinal/perirhinal cortex. This finding was extended to the spatial domain by contrasting activity when participants were presented with mismatches either in terms of sequence (with spatial location repeated) or spatial location (with temporal sequence repeated, Kumaran \& Maguire, 2007). Similar to the original study, greater activity was seen in the left hippocampus when either location or sequence mismatched the first presentation. These authors interpret their findings as support for a view in which the left hippocampus generates novelty signals when sensory input is discrepant from stored representations of previous experience (see also Gray, 1982; Kumaran \& Maguire, 2007, 2009 for more details). It remains to be addressed, however, whether these mismatch effects are always hippocampally-dependent or whether mismatch for other types of information beyond temporal and spatial, such as mismatches in ambiguous object features, would be dependent upon representations stored within the perirhinal cortex (as predicted by our theoretical position; see Duncan, Curtis, \& Davachi (2009) for evidence of perirhinal activity during an object mismatch).

In addition, there is also preliminary evidence that language itself may be impaired in amnesia (Knott \& Marslen-Wilson, 2001; MacKay, 2001, 2006; MacKay, James, Taylor, \& Marian, 2007; MacKay, Stewart, \& Burke, 1998a, 1998b; although see Kensinger, Ullman, \& Corkin, 2001). MacKay and colleagues reported impairments in a single amnesic case (HM), including poor semantic ambiguity detection, generation of impossible interpretations, free associations between words, lexical decision, meaning-definition tasks, reading, producing and comprehending unfamiliar novel sentences (James \& MacKay, 2001; MacKay, et al., 1998; MacKay \& James, 2002; MacKay, James, \& Hadley, 2007; MacKay, James, Taylor, et al., 2007; MacKay et al., 1998a; MacKay et al., 2007b, see also MacKay \& James, 2009, for deficits in the domain of visual perception). MacKay and colleagues propose that MTL damage affects binding processes necessary for integrating information that has never previously been activated together, but does not impair binding of information that has co-occurred previously and for which there is already existing connections. This account could provide an explanation for the difficulties that amnesic individuals show in memory for word lists and prose passages: while the lexical items that comprise these stimuli are often familiar and have semantic meaning (in the sense that they were acquired many years prior to brain damage), their co-occurrence in the lists or sentences are novel, and consequently it is not possible for amnesic individuals to create the rapid conjunctive associative connections required to form representations of this novel entity. This explanation parallels that put forward earlier in this review in the context of perceptual representations for objects and spatial environments, and highlights the need for further studies of language beyond the single case (HM) reported by MacKay and his collaborators in order to identify whether these impairments generalise to other individuals with MTL damage or were specific to HM.

\subsection{Summary}

In summary, it has long been taken for granted that, “... .memory is a distinct cerebral function, separable from other perceptual and cognitive abilities" (Squire, 2009, p. 2). This review reveals an increasing body of cross-species and cross-methodological evidence to the contrary. While there are significant challenges ahead, both for EMA and purely mnemonic views of the MTL, debates regarding these issues will lead to more dynamic and interactive models of human memory and a better understanding of the genesis of memory disorders.

\section{Acknowledgements}

We would like to thank the wonderful colleagues who have contributed to the work and ideas discussed in this review, in particular Stephan Bandelow, Mark Buckley, Tim Bussey, Rhys Davies, Audrey Duarte, Tina Emery, David Gaffan, Kate Gough, Rik Henson, Narinder Kapur, Matthew Mundy, Betsy Murray, Lisa Saksida, Vicky Scahill, Hugo Spiers and Karen Taylor. We are also grateful to Tim Bussey, Betsy Murray, Lisa Saksida, Charan Ranganath, Deborah Hannula and Yael Shrager for providing figures and data from their published work, and to Tim Smith, Karen Taylor and Andrew Lawrence for comments on the manuscript and theoretical issues. We also extend our thanks to the patients and volunteers who have given so generously of their time to further our understanding of the medial temporal lobe, in particular the case who provided the transcript described at the beginning of this review. Finally, we thank the reviewers of this manuscript, in particular Morris Moscovitch, for their helpful comments regarding the experiments, data and theoretical model discussed in the review. The authors received financial support for this work from the Medical Research Council, UK (all authors), the Alzheimer's Research Trust (KG and AL), Peterhouse, Cambridge (MB), the Wellcome Trust (\#082315, AL) and the Wales Institute of Cognitive Neuroscience, established via a grant from the Welsh Assembly Government to Cardiff, Bangor and Swansea Universities (KG).

\section{References}

Aggleton, J. P., \& Brown, M. W. (1999). Episodic memory, amnesia, and the hippocampal-anterior thalamic axis. Behavioural Brain Sciences, 22, 425-444 [discussion 444-489] 
Aggleton, J.P., \& Brown, M.W.(2005). Contrasting hippocampal and perirhinal cortex function using immediate early gene imaging. Quarterly Journal of Experimental Psychology B, 58, 218-233.

Aggleton, J. P., \& Brown, M. W. (2006). Interleaving brain systems for episodic and recognition memory. Trends in Cognitive Science, 10, 455-463.

Aggleton, J. P., \& Shaw, C. (1996). Amnesia and recognition memory: A re-analysis of psychometric data. Neuropsychologia, 34, 51-62.

Aggleton, J. P., Hunt, P. R., \& Rawlins, J. N. (1986). The effects of hippocampal lesions upon spatial and non-spatial tests of working memory. Behavioural Brain Research, 19, 133-146.

Aggleton, J. P., Shaw, C., \& Gaffan, E. A. (1992). The performance of postencephalitic amnesic subjects on two behavioural tests of memory: Concurrent discrimination learning and delayed matching-to-sample. Cortex, 28, 359-372.

Aggleton, J. P., Vann, S. D., Denby, C., Dix, S., Mayes, A. R., Roberts, N., et al. (2005). Sparing of the familiarity component of recognition memory in a patient with hippocampal pathology. Neuropsychologia, 43, 1810-1823.

Aggleton, J. P., Sanderson, D. J., \& Pearce, J. M. (2007). Structural learning and the hippocampus. Hippocampus, 17, 723-734.

Alverson, H. (1994). Semantics and experience: Universal metaphors of time in English, Mandarin, Hindi, and Sesotho. Baltimore: John Hopkins University Press.

Awipi, T., \& Davachi, L. (2008). Content-specific source encoding in the human medial temporal lobe. Journal of Experimental Psychology: Learning, Memory and Cognition, 34, 769-779.

Baddeley, A., Vargha-Khadem, F., \& Mishkin, M. (2001). Preserved recognition in a case of developmental amnesia: Implications for the acquisition of semantic memory? Journal of Cognitive Neuroscience, 13, 357-369.

Bar, M., Aminoff, E., \& Schacter, D. L. (2008). Scenes unseen: The parahippocampal cortex intrinsically subserves contextual associations, not scenes or places per se. Journal of Neuroscience, 28, 8539-8544.

Barense, M. D., Bussey, T. J., Lee, A. C. H., Rogers, T. T., Davies, R. R., Saksida, L. M., et al. (2005). Functional specialization in the human medial temporal lobe. Journal of Neuroscience, 25, 10239-10246.

Barense, M. D., Gaffan, D., \& Graham, K. S. (2007). The medial temporal lobe processes online representations of complex objects. Neuropsychologia, 45, 2963-2974.

Barense, M. D., Henson, R. N. A., Schwarzbauer, C., \& Graham, K. S. (2008). Temporal lobe activity during complex discriminations of familiar and novel objects and faces. In Annual meeting of the Society for Neuroscience Washington DC.

Barense, M. D., Henson, R. N. A., Lee, A. C. H., \& Graham, K. S. (2009). Medial temporal lobe activity during complex discriminations of faces, objects and scenes: Effects of viewpoint. Hippocampus [Epub June 4].

Barense, M.D., Rogers, T.T., Bussey, T.J., Saksida, L. \& Graham, K.S. (in press). Influence of conceptual knowledge of visual object discrimination: Insights from semantic dementia and MTL amnesia. Cerebral Cortex.

Bartko, S. J., Winters, B. D., Cowell, R. A., Saksida, L. M., \& Bussey, T. J. (2007a). Perceptual functions of perirhinal cortex in rats: Zero-delay object recognition and simultaneous oddity discriminations. Journal of Neuroscience, 27, 25482549.

Bartko, S. J., Winters, B. D., Cowell, R. A., Saksida, L. M., \& Bussey, T. J. (2007b) Perirhinal cortex resolves feature ambiguity in configural object recognition and perceptual oddity tasks. Learning and Memory, 14, 821-832.

Baxter, M. G. (2009). Involvement of medial temporal lobe structures in memory and perception. Neuron, 61, 667-677.

Bayley, P. J., Hopkins, R. O., \& Squire, L. R. (2003). Successful recollection of remote autobiographical memories by amnesic patients with medial temporal lobe lesions. Neuron, $38,135-144$

Bird, C. M., \& Burgess, N. (2008a). The hippocampus and memory: Insights from spatial processing. Nature Reviews Neuroscience, 9, 182-194.

Bird, C. M., \& Burgess, N. (2008b). The hippocampus supports recognition memory for familiar words but not unfamiliar faces. Current Biology, 18, 1932-1936.

Bird, C. M., Shallice, T., \& Cipolotti, L. (2007). Fractionation of memory in medial temporal lobe amnesia. Neuropsychologia, 45, 1160-1171.

Bird, C. M., Vargha-Khadem, F., \& Burgess. (2008). Impaired memory for scenes but not faces in developmental hippocampal amnesia: A case study. Neuropsychologia, 46, 1050-1059.

Bohbot, V. D., Kalina, M., Stepankova, J., Spackova, N., Petrides, M., \& Nadel, L. (1998). Spatial memory deficits in patients with lesions to the right hippocampus and to the right parahippocampal cortex. Neuropsychologia, 36, 1217-1238.

Bohbot, V. D., Iaria, G., \& Petrides, M. (2004). Hippocampal function and spatial memory: Evidence from functional neuroimaging in healthy participants and performance of patients with medial temporal lobe resections. Neuropsychology, $18,418-425$

Boroditsky, L. (2000). Metaphoric structuring: Understanding time through spatial metaphors. Cognition, 75, 1-28.

Boroditsky, L. (2001). Does language shape thought? Mandarin and English speakers' conceptions of time. Cognitive Psychology, 43, 1-22.

Bowerman, M. (1983). Hidden meanings: The role of covert conceptual structures in children's development of language. In D.R. Rogers \& J.A. Sloboda (Eds.), The acquisition of symbolic skills. New York: Plenum.

Bowles, B., Crupi, C., Mirasattari, S. M., Pigott, S. E., Parrent, A. G., Pruessner, J. C., et al. (2007). Impaired familiarity with preserved recollection after anterior temporallobe resection that spares the hippocampus. Proceedings of the National Academy of Sciences (USA), 104, 16382-16387.

Brandt, K. R., Gardiner, J. M., Vargha-Khadem, F., Baddeley, A. D., \& Mishkin, M. (2008). Impairment of recollection but not familiarity in a case of developmental amnesia. Neurocase, 17, 1-6.
Bright, P., Moss, H. E., Stamatakis, E. A., \& Tyler, L. K. (2005). The anatomy of object processing: The role of anteromedial temporal cortex. Quarterly Journal of Experimental Psychology B, 58, 361-377.

Bright, P., Buckman, J., Fradera, A., Yoshimasu, H., Colchester, A. C., \& Kopelman, M. D. (2006). Retrograde amnesia in patients with hippocampal, medial temporal, temporal lobe, or frontal pathology. Learning and Memory, 13, 545-557.

Brockmole, J. R., \& Henderson, J. M. (2006). Recognition and attention guidance during contextual cueing in real-world scenes: Evidence from eye movements. Quarterly Journal of Experimental Psychology, 59, 1177-1187.

Brown, M. W., \& Aggleton, J. P. (2001). Recognition memory: What are the roles of the perirhinal cortex and hippocampus? Nature Reviews Neuroscience, 2, 51-61.

Brown, M. W., \& Xiang, J. Z. (1998). Recognition memory: Neuronal substrates of the judgement of prior occurrence. Progress in Neurobiology, 55, 149-189.

Brown, M. W., Wilson, F. A., \& Riches, I. P. (1987). Neuronal evidence that inferomedial temporal cortex is more important than hippocampus in certain processes underlying recognition memory. Brain Research, 409, 158-162.

Buckley, M. J. (2005). The role of the perirhinal cortex and hippocampus in learning, memory, and perception. Quarterly Journal of Experimental Psychology B, 58 , 246-268.

Buckley, M. J., \& Gaffan, D. (1997). Impairment of visual object-discrimination learning after perirhinal cortex ablation. Behavioral Neuroscience, 111, 467-475.

Buckley, M. J., \& Gaffan, D. (1998a). Perirhinal cortex ablation impairs configural learning and paired-associate learning equally. Neuropsychologia, 36, $535-546$.

Buckley, M. J., \& Gaffan, D. (1998b). Perirhinal cortex ablation impairs visual object identification. Journal of Neuroscience, 18, 2268-2275.

Buckley, M. J., \& Gaffan, D. (2006). Perirhinal cortical contributions to object perception. Trends in Cognitive Sciences, 10, 100-107.

Buckley, M. J., Gaffan, D., \& Murray, E. A. (1997). Functional double dissociation between two inferior temporal cortical areas: Perirhinal cortex versus middle temporal gyrus. Journal of Neurophysiology, 77, 587-598.

Buckley, M. J., Booth, M. C., Rolls, E. T., \& Gaffan, D. (2001). Selective perceptual impairments after perirhinal cortex ablation. Journal of Neuroscience, 21, 9824-9836.

Buckley, M. J., Charles, D. P., Browning, P. G., \& Gaffan, D. (2004). Learning and retrieval of concurrently presented spatial discrimination tasks: Role of the fornix. Behavioural Neuroscience, 118, 138-149.

Buffalo, E. D., Reber, P. J., \& Squire, L. R. (1998). The human perirhinal cortex and recognition memory. Hippocampus, 8, 330-339.

Buffalo, E. A., Bellgowan, P. S., \& Martin, A. (2006). Distinct roles for medial temporal lobe structures in memory for objects and their locations. Learning and Memory, 13, 638-643.

Burgess, N., Maguire, E. A., \& O'Keefe, J. (2002). The human hippocampus and spatial and episodic memory. Neuron, 35, 625-641.

Bussey, T. J., \& Saksida, L. M. (2005). Object memory and perception in the medial temporal lobe: An alternative approach. Current Opinion in Neurobiology, 15, 730-737.

Bussey, T. J., \& Saksida, L. M. (2007). Memory, perception and the ventral visualperirhinal-hippocampal stream: Thinking outside the boxes. Hippocampus, 17 , 898-908.

Bussey, T. J., Saksida, L. M., \& Murray, E. A. (2002). Perirhinal cortex resolves feature ambiguity in complex visual discriminations. European Journal of Neuroscience, $15,365-374$.

Bussey, T. J., Saksida, L. M., \& Murray, E. A. (2003). Impairments in visual discrimination after perirhinal cortex lesions: Testing 'declarative' vs. 'perceptual-mnemonic' views of perirhinal cortex function. European Journal of Neuroscience, 17, 649-660.

Bussey, T. J., Saksida, L. M., \& Murray, E. A. (2005). The perceptual-mnemonic/feature conjunction model of perirhinal cortex function. Quarterly Journal of Experimental Psychology B, 58, 269-282.

Buzsaki, G. (2002). Large-scale recording of neuronal ensembles. Nature Neuroscience, 7, 446-451.

Cansino, S., Maquet, P., Dolan, R. J., \& Rugg, M. D. (2002). Brain activity underlying encoding and retrieval of source memory. Cerebral Cortex, 12, 1048-1056.

Carlesimo, G. A., Serra, L., Fadda, L., Cherubini, A., Bozzali, M., \& Caltagirone, C. (2007). Bilateral damage to the mammillo-thalamic tract impairs recollection but not familiarity in the recognition process: A single case investigation. Neuropsychologia, 45, 2467-2479.

Casasanto, D., \& Boroditsky, L. (2008). Time in the mind: Using space to think about time. Cognition, 106, 579-593.

Charles, D. P., Gaffan, D., \& Buckley, M. J. (2004). Impaired recency judgments and intact novelty judgments after fornix transection in monkeys. Journal of Neuroscience, 24, 2037-2044.

Chun, M. M., \& Jiang, Y. (1998). Contextual cueing. Implicit learning and memory for visual context guides spatial attention. Cognitive Psychology, 36, 28-71.

Cipolotti, L., \& Maguire, E. A. (2003). A combined neuropsychological and neuroimaging study of topographical and non-verbal memory in semantic dementia. Neuropsychologia, 41, 1148-1159.

Cipolotti, L., Bird, C., Good, T., Macmanus, D., Rudge, P., \& Shallice, T. (2006). Recollection and familiarity in dense hippocampal amnesia: A case study. Neuropsychologia, 44, 489-506.

Cohen, N. J., \& Eichenbaum, H. (1993). Memory, amnesia, and the hippocampal system. Massachusetts: MIT Press.

Cohen, N. J., Poldrack, R. A., \& Eichenbaum, H. (1997). Memory for items and memory for relations in the procedural/declarative memory framework. Memory, 5 131-178. 
Cohen, N. J., Ryan, J., Hunt, C., Romine, L., Wszalek, T., \& Nash, C. (1999). Hippocampal system and declarative (relational) memory: Summarizing the data from functional neuroimaging studies. Hippocampus, 9, 83-98.

Corkin, S. (1968). Aquisition of motor skill after bilateral medial temporal excision. Neuropsychologia, 4, 255-265.

Corkin, S., Amaral, D. G., Gonzalez, R. G., Johnson, K. A., \& Hyman, B. T. (1997). H.M.'s medial temporal lobe lesion: Findings from magnetic resonance imaging.Journal of Neuroscience, 17, 3964-3979.

Correll, R. E., \& Scoville, W. B. (1965a). Effects of medial temporal lesions on visual discrimination performance. Journal of Comparative and Physiological Psychology, $60,175-181$.

Correll, R. E., \& Scoville, W. B. (1965b). Performance of delayed match following lesions of medial temporal lobe structures. Journal of Comparative and Physiological Psychology, 60, 360-367.

Cowell, R. A., Bussey, T. J., \& Saksida, L. M. (2006). Why does brain damage impair memory? A connectionist model of object recognition memory in perirhinal cortex. Journal of Neuroscience, 26, 12186-12197.

Cumming, T. B., Patterson, K., Verfaellie, M., \& Graham, K. S. (2006). One bird with two stones: Letter-by-letter reading in pure alexia and semantic dementia. Cognitive Neuropsychology, 23, 1130-1161.

Damasio, A. R., Eslinger, P., Damasio, H., Van Hoesen, G. W., \& Cornell, S. (1985). Multimodal amnesic syndrome following bilateral temporal and basal forebrain damage. Archives of Neurology, 42, 252-259.

Davachi, L., \& Wagner, A. D. (2002). Hippocampal contributions to episodic encoding: Insights from relational and item-based learning. Journal of Neurophysiology, 88, 982-990.

Davachi, L., Mitchell, J. P., \& Wagner, A. D. (2003). Multiple routes to memory: Distinct medial temporal lobe processes build item and source memories. Proceedings of the National Academy of Sciences USA, 100, 2157-2162.

Davies, R. R., Graham, K. S., Xuereb, J. H., Williams, G. B., \& Hodges, J. R. (2004). The human perirhinal cortex and semantic memory. European Journal of Neuroscience, 20, 2441-2446.

DeJong, R. N. (1973). The hippocampus and its role in memory. Clinical manifestations and theoretical considerations. Journal of Neurological Sciences, 19, 73-83.

Devlin, J. T., \& Price, C. J. (2007). Perirhinal contributions to human visual perception. Current Biology, 17, 1484-1488.

Diana, R. A., Yonelinas, A. P., \& Ranganath, C. (2007). Imaging recollection and familiarity in the medial temporal lobe: A three-component model. Trends in Cognitive Sciences, 11, 379-386.

Diana, R. A., Yonelinas, A. P., \& Ranganath, C. (2008). High-resolution multi-voxel pattern analysis of category selectivity in the medial temporal lobes. Hippocampus, $18,536-541$.

Diana, R. A., Yonelinas, A. P., \& Ranganath, C. (2009). Medial temporal lobe activity during source retrieval reflects information type, not memory strength. Journal of Cognitive Neuroscience [Epub Aug 24].

Dragoi, G., \& Buzsaki, G. (2006). Temporal encoding of place sequences by hippocampal cell assemblies. Neuron, 50, 145-157.

Duarte, A., Henson, R.N.A., \& Graham, K.S. (submitted for publication). Stimulus content and the neural correlates of item and source memory. Journal of Cognitive Neuroscience.

Duncan, J. (2001). An adaptive coding model of neural function in prefrontal cortex. Nature Reviews Neuroscience, 2, 820-829.

Duncan, J., Curtis, C., \& Davachi, L. (2009). Distinct memory signatures in the hippocampus: Intentional states distinguish match and mismatch enhancement signals. Journal of Neuroscience, 29, 131-139.

Eacott, M. J., \& Gaffan, E. A. (2005). The roles of perirhinal cortex, postrhinal cortex, and the fornix in memory for objects, contexts, and events in the rat. Quarterly Journal of Experimental Psychology B, 58, 202-217.

Eacott, M. J., Gaffan, D., \& Murray, E. A. (1994). Preserved recognition memory for small sets, and impaired stimulus identification for large sets, following rhinal cortex ablations in monkeys. European Journal of Neuroscience, 6, 1466-1478.

Easton, A., \& Gaffan, D. (2000). Comparison of perirhinal cortex ablation and crossed unilateral lesions of the medial forebrain bundle from the inferior temporal cortex in the rhesus monkey: Effects on learning and retrieval. Behavioural Neuroscience, 114, 1041-1057.

Easton, A., \& Gaffan, D. (2001). Crossed unilateral lesions of the medial forebrain bundle and either inferior temporal or frontal cortex impair object-reward association learning in Rhesus monkeys. Neuropsychologia, 39, 71-82.

Easton, A., Parker, A., \& Gaffan, D. (2001). Crossed unilateral lesions of medial forebrain bundle and either inferior temporal or frontal cortex impair object recognition in Rhesus monkeys. Behavioural Brain Research, 121, 1-10.

Eichenbaum, H. (2000a). A cortical-hippocampal system for declarative memory. Nature Reviews Neuroscience, 1, 41-50.

Eichenbaum, H. (2000b). Hippocampus: Mapping or memory? Current Biology, 10, R785-787.

Eichenbaum, H. (2004). Hippocampus: Cognitive processes and neural representations that underlie declarative memory. Neuron, 44, 109-120.

Eichenbaum, H., \& Cohen, N. J. (2001). From conditioning to conscious recollection: Memory systems of the brain. New York, NY: Oxford University Press.

Eichenbaum, H., Otto, T., \& Cohen, N. J. (1994). Two functional components of the hippocampal memory system. Behavioural Brain Sciences, 17, 449-518.

Eichenbaum, H., Dudchenko, P., Wood, E., Shapiro, M., \& Tanila, H. (1999). The hippocampus, memory, and place cells: It is spatial memory or a memory space? Neuron, 23, 209-226.

Eichenbaum, H., Yonelinas, A. P., \& Ranganath, C. (2007). The medial temporal lobe and recognition memory. Annual Reviews in Neuroscience, 30, 123-152.
Ekstrom, A. D., Kahana, M. J., Caplan, J. B., Fields, T. A., Isham, E. A., Newman, E. L., et al. (2003). Cellular networks underlying human spatial navigation. Nature, 425 , $184-188$.

Eldridge, L. L., Knowlton, B. J., Furmanski, C. S., Bookheimer, S. Y., \& Engel, S. A. (2000) Remembering episodes: A selective role for the hippocampus during retrieval. Nature Neuroscience, 3, 1149-1152.

Ennaceur, A., \& Aggleton, J. P. (1997). The effects of neurotoxic lesions of the perirhinal cortex combined to fornix transection on object recognition memory in the rat. Behavioural Brain Research, 88, 181-193.

Ennaceur, A., Neave, N., \& Aggleton, J. P. (1996). Neurotoxic lesions of the perirhina cortex do not mimic the behavioural effects of fornix transection in the rat Behavioural Brain Research, 80, 9-25.

Epstein, R. (2008). Parahippocampal and retrosplenial contributions to human spatial navigation. Trends in Cognitive Sciences, 12, 388-396.

Epstein, R., Graham, K. S., \& Downing, P. E. (2003). Viewpoint-specific scene representations in human parahippocampal cortex. Neuron, 37, 865-876.

Ewbank, M. P., Schluppeck, D., \& Andrews, T. J. (2005). fMR-adaptation reveals a distributed representation of inanimate objects and places in human visual cortex. Neuroimage, 28, 268-279.

Feigenbaum, J. D., \& Morris, R. G. (2004). Allocentric versus egocentric memory after unilateral temporal lobectomy in humans. Neuropsychology, 18, 462-472.

Fortin, N. J., Agster, K. L., \& Eichenbaum, H. B. (2002). Critical role of the hippocampus in memory for sequences of events. Nature Neuroscience, 5, 458-462.

Gaffan, D. (1994). Scene-specific memory for objects: A model of episodic memory impairment in monkeys with fornix transection. Journal of Cognitive Neuroscience, 6, 305-320.

Gaffan, D. (2001). What is a memory system? Horel's critique revisited. Behaviora Brain Research, 127, 5-11.

Gaffan, D. (2002). Against memory systems. Philosophical Transactions of the Royal Society London. B Biological Sciences, 357, 1111-1121.

Gaffan, D., Parker, A., \& Easton, A. (2001). Dense amnesia in the monkey after transection of fornix, amygdala and anterior temporal stem. Neuropsychologia, 39, 51-70.

Gardiner, J. M. (1988). Recognition failures and free-recall failures: Implication for the relation between recall and recognition. Memory and Cognition, 16, 446-451.

Giovanello, K. S., \& Verfaellie, M. (2001). The relationship between recall and recognition in amnesia: Effects of matching recognition between patients with amnesia and controls. Neuropsychology, 15, 444-451.

Giovanello, K. S., Verfaellie, M., \& Keane, M. M. (2003). Disproportionate deficit in associative recognition relative to item recognition in global amnesia. Cognitive Affective and Behavioural Neuroscience, 3, 186-194.

Giovanello, K. S., Schnyer, D. M., \& Verfaellie, M. (2004). A critical role for the anterior hippocampus in relational memory: Evidence from an fMRI study comparing associative and item recognition. Hippocampus, 14, 5-8.

Gold, J. J., Hopkins, R. O., \& Squire, L. R. (2006). Single-item memory, associative memory, and the human hippocampus. Learning and Memory, 13, 644-649.

Graham, K. S., Simons, J. S., Pratt, K. H., Patterson, K., \& Hodges, J. R. (2000). Insights from semantic dementia on the relationship between episodic and semantic memory. Neuropsychologia, 38, 313-324.

Graham, K. S., Patterson, K., Powis, J., Drake, J., \& Hodges, J. R. (2002). Multiple inputs to episodic memory: Words tell another story. Neuropsychology, 16, 380-389.

Graham, K. S., Scahill, V. L., Hornberger, M., Barense, M. D., Lee, A. C., Bussey, T. J., et al. (2006). Abnormal categorization and perceptual learning in patients with hippocampal damage. Journal of Neuroscience, 26, 7547-7554.

Graham, K. S., Lee, A. C. H., \& Barense, M. D. (2007). Impairments in visual discrimination in amnesia: Implications for theories of the role of medial temporal lobe regions in human memory. European Journal of Cognitive Psychology, 20, 655-596.

Graham, K.S., Lee, A.C.H., \& Barense, M.D. (2008). Memory and perceptual impairments in amnesia and dementia. In E. Dere, A. Easton, J. Huston, \& L. Nadel (Eds.) Handbook of Behavioural Neuroscience, Vol 18. Episodic Memory (pp. 483-501).

Gray, J. A. (1982). The neuropsychology of anxiety: An enquiry into the functions of the septo-hippocampal system. Oxford: Oxford University Press.

Hamann, S. B., \& Squire, L. R. (1997). Intact perceptual memory in the absence of conscious memory. Behavioural Neuroscience, 111, 850-854.

Hampton, R. R. (2005). Monkey perirhinal cortex is critical for visual memory, but not for visual perception: Reexamination of the behavioural evidence from monkeys. Quarterly Journal of Experimental Psychology B, 58, 283-299.

Hampton, R. R., \& Murray, E. A. (2002). Learning of discriminations is impaired, but generalization to altered views is intact, in monkeys (Macaca mulatta) with perirhinal cortex removal. Behavioural Neuroscience, 116, 363-377.

Hannula, D. E., \& Ranganath, C. (2008). Medial temporal lobe activity predicts successful relational memory binding. Journal of Neuroscience, 28, 116-124.

Hannula, D. E., Tranel, D., \& Cohen, N. J. (2006). The long and the short of it: Relational memory impairments in amnesia, even at short lags. Journal of Neuroscience, 26 8352-8359.

Harrison, S., \& Tong, F. (2009). Decoding reveals the contents of visual working memory in early visual areas. Nature, $458,632-635$

Hartley, T., Bird, C. M., Chan, D., Cipolotti, L., Husain, M., Vargha-Khadem, F., et al. (2007). The hippocampus is required for short-term topographical memory in humans. Hippocampus, 17, 34-48.

Haskins, A. L., Yonelinas, A. P., Quamme, J. R., \& Ranganath, C. (2008). Perirhinal cortex supports encoding and familiarity-based recognition of novel associations. Neuron, 59, 554-560.

Henderson, J. M. (2003). Human gaze control in real-world scene perception. Trends in Cognitive Sciences, 7, 498-504. 
Henderson, J. M., \& Hollingworth, A. (1999). High-level scene perception. Annual Reviews in Psychology, 50, 243-271.

Henderson, J. M., Weeks, P. A., Jr., \& Hollingworth, A. (1999). Effects of semantic consistency on eye movements during scene viewing. Journal of Experimental Psychology: Human Perception and Performance, 25, 210-228.

Henson, R., Cansino, S., Herron, J. E., Robb, W. G., \& Rugg, M. D. (2003). A familiarity signal in human anterior medial temporal cortex? Hippocampus, 13, 301-304.

Hirst, W., Johnson, M. K., Kim, J. K., Phelps, E. A., Risse, G., \& Volpe, B. T. (1986). Recognition and recall in amnesics. Journal of Experimental Psychology: Learning, Memory and Cognition, 12, 445-451.

Hirst, W., Johnson, M. K., Phelps, E. A., \& Volpe, B. T. (1988). More on recognition and recall in amnesics. Journal of Experimental Psychology: Learning, Memory and Cognition, 14, 758-762.

Holdstock, J. S., Shaw, C., \& Aggleton, J. P. (1995). The performance of amnesic subjects on tests of delayed matching-to-sample and delayed matching-to-position. Neuropsychologia, 33, 1583-1596.

Holdstock, J. S., Gutnikov, S. A., Gaffan, D., \& Mayes, A. R. (2000). Perceptual and mnemonic matching-to-sample in humans: Contributions of the hippocampus, perirhinal and other medial temporal lobe cortices. Cortex, 36, 301-322.

Holdstock, J. S., Mayes, A. R., Cezayirli, E., Isaac, C. L., Aggleton, J. P., \& Roberts, N. (2000). A comparison of egocentric and allocentric spatial memory in a patient with selective hippocampal damage. Neuropsychologia, 38, 410-425.

Holdstock, J. S., Mayes, A. R., Roberts, N., Cezayirli, E., Isaac, C. L., O’Reilly, R. C., et al. (2002). Under what conditions is recognition spared relative to recall after selective hippocampal damage in humans? Hippocampus, 12, 341-351.

Hollingworth, A. (2004). Constructing visual representations of natural scenes: The roles of short- and long-term visual memory. Journal of Experimental Psychology: Human Perception and Performance, 30, 519-537.

Hollingworth, A. (2005). The relationship between online visual representation of a scene and long-term scene memory. Journal of Experimental Psychology: Learning, Memory and Cognition, 31, 396-411.

Hollingworth, A. (2007). Object-position binding in visual memory for natural scenes and object arrays. Journal of Experimental Psychology: Human Perception and Performance, 33, 31-47.

Hollingworth, A., \& Henderson, J. M. (2002). Accurate visual memory for previously attended objects in natural scenes. Journal of Experimental Psychology: Human Perception and Performance, 28, 113-136.

Hollingworth, A., Williams, C. C., \& Henderson, J. M. (2001). To see and remember: Visually specific information is retained in memory from previously attended objects in natural scenes. Psychonomic Bulletin and Review, 8, 761-768.

Horel, J. A. (1978). The neuroanatomy of amnesia. A critique of the hippocampal memory hypothesis. Brain, 101, 403-445.

James, L. E., \& MacKay, D. G. (2001). H.M., word knowledge and aging: Support for a new theory of long-term retrograde amnesia. Psychological Science, 12, 485-492.

Keane, M. M., Gabrieli, J. D., Mapstone, H. C., Johnson, K. A., \& Corkin, S. (1995). Double dissociation of memory capacities after bilateral occipital-lobe or medial temporal-lobe lesions. Brain, 118, 1129-1148.

Kensinger, E. A., \& Schacter, D. L. (2006). Amygdala activity is associated with successful encoding of item, but not source, information for positive and negative stimuli. Journal of Neuroscience, 26, 2564-2570.

Kensinger, E. A., Ullman, M. T., \& Corkin, S. (2001). Bilateral medial temporal lobe damage does not affect lexical or grammatical processing: Evidence from amnesic patient H.M. Hippocampus, 11, 347-360.

King, J. A., Burgess, N., Hartley, T., Vargha-Khadem, F., \& O’Keefe, J. (2002). Human hippocampus and viewpoint dependence in spatial memory. Hippocampus, 12 , 811-820.

King, J. A., Trinkler, I., Hartley, T., Vargha-Khadem, F., \& Burgess, N. (2004). The hippocampal role in spatial memory and the familiarity-recollection distinction: A case study. Neuropsychology, 18, 405-417.

Kirchhoff, B. A., Wagner, A. D., Maril, A., \& Stern, C. E. (2000). Prefrontal-temporal circuitry for episodic encoding and subsequent memory. Journal of Neuroscience, 20, 6173-6180.

Kirwan, C. B., Wixted, J. T., \& Squire, L. R. (2008). Activity in the medial temporal lobe predicts memory strength, whereas activity in the prefrontal cortex predicts recollection. Journal of Neuroscience, 28, 10541-10548.

Knott, R., \& Marslen-Wilson, W. (2001). Does the medial temporal lobe bind phonological memories? Journal of Cognitive Neuroscience, 13, 593-609.

Knowlton, B. J., \& Squire, L. R. (1993). The learning of categories: Parallel brain systems for item memory and category knowledge. Science, 10, 1747-1749.

Konkel, A., Warren, D. E., Duff, M. C., Tranel, D. N., \& Cohen, N. J. (2008). Hippocampal amnesia impairs all manner of relational memory. Frontiers in Human Neuroscience, $2,1-15$.

Kumaran, D., \& Maguire, E. A. (2005). The human hippocampus: Cognitive maps or relational memory? Journal of Neuroscience, 25, 7254-7259.

Kumaran, D., \& Maguire, E. A. (2006). An unexpected sequence of events: Mismatch detection in the human hippocampus. PLoS Biology, 4, 2372-2381.

Kumaran, D., \& Maguire, E. A. (2007). Match mismatch processes underlie human hippocampal responses to associative novelty. Journal of Neuroscience, 27, 8517-8524.

Kumaran, D., \& Maguire, E. A. (2009). Novelty signals: A window into hippocampal information processing. Trends in Cognitive Science, 13, 47-54.

Lee, A.C.H. \& Rudebeck, S.R. (2009). Investigating the interaction between spatial perception and working memory in the human medial temporal lobe. Journal of Cognitive Neuroscience [E-pub Nov 19].

Lee, A. C. H., Barense, M. D., \& Graham, K. S. (2005). The contribution of the human medial temporal lobe to perception: Bridging the gap between ani- mal and human studies. Quarterly Journal of Experimental Psychology B, 58, 300-325.

Lee, A. C. H., Buckley, M. J., Pegman, S. J., Spiers, H., Scahill, V. L., Gaffan, D., et al. (2005). Specialization in the medial temporal lobe for processing of objects and scenes. Hippocampus, 15, 782-797.

Lee, A. C. H., Bussey, T. J., Murray, E. A., Saksida, L. M., Epstein, R. A., Kapur, N., et al. (2005). Perceptual deficits in amnesia: Challenging the medial temporal lobe 'mnemonic' view. Neuropsychologia, 43, 1-11.

Lee, A. C. H., Bandelow, S., Schwarzbauer, C., Henson, R., \& Graham, K. S. (2006). Perirhinal cortex activity during visual object discrimination: An event-related fMRI study. Neuroimage, 33, 362-373.

Lee, A. C. H., Buckley, M. J., Gaffan, D., Emery, T., Hodges, J. R., \& Graham, K. S. (2006). Differentiating the roles of the hippocampus and perirhinal cortex in processes beyond long-term declarative memory: A double dissociation in dementia. Journal of Neuroscience, 26, 5198-5203.

Lee, A. C. H., Levi, N., Davies, R. R., Hodges, J. R., \& Graham, K. S. (2007). Differing profiles of face and scene discrimination deficits in semantic dementia and Alzheimer's disease. Neuropsychologia, 45, 2135-2146.

Lee, A. C. H., Gough, K., Davies, R. R., Barense, M. D., \& Graham, K. S. (2008). The medial temporal lobe and processes beyond long-term memory: A volumetric MRI study. Society for Neuroscience, 785.1.

Lee, A. C. H., Scahill, V. L., \& Graham, K. S. (2008). Activating the medial temporal lobe during oddity judgement for faces and scenes. Cerebral Cortex, 18, 683-696.

Lehky, S. R., \& Tanaka, K. (2007). Enhancement of object representations in primate perirhinal cortex during a visual working-memory task. Journal of Neurophysiology, 97, 1298-1310.

Levy, D. A., Shrager, Y., \& Squire, L. R. (2005). Intact visual discrimination of complex and feature-ambiguous stimuli in the absence of perirhinal cortex. Learning and Memory, 12, 61-66.

Litman, L., Awipi, T., \& Davachi, L. (2009). Category-specificity in the human medial temporal cortex. Hippocampus, 19, 308-319.

Lopez-Aranda, M. F., Lopez-Tellez, J. F., Navarro-Lobato, I., Masmudi-Martin, M., Gutierrez, A., \& Khan, Z. U. (2009). Role of layer 6 of V2 visual cortex in objectrecognition memory. Science, 325, 87-89.

MacKay, D. G. (2001). A tale of two paradigms or metatheoretical approaches to cognitive neuropsychology: Did Schmolck, Stefanacci, and Squire (2000) show that hippocampal lesions only impair memory, whereas adjacent (extrahippocampal) lesions impair detection and explanation of sentence ambiguity. Brain and Language, 78, 265-272.

MacKay, D. G. (2006). Aging, memory and language in amnesic H.M. Hippocampus, $16,491-494$.

MacKay, D. G., \& James, L. E. (2002). Aging, retrograde amnesia, and the binding problem for phonology and orthography: A longitudinal study of 'hippocampal amnesia' H.M. Aging. Neuropsychology and Cognition, 9, 298-333.

MacKay, D. G., \& James, L. E. (2009). Visual cognition in amnesic H.M.: Selective deficits on the What's-wrong-here and Hidden-Figure tasks. Journal of Clinical and Experimental Neuropsychology [E-pub January 14th].

MacKay, D. G., Stewart, R., \& Burke, D. M. (1998a). H.M. revisited: Relations between language comprehension, memory and the hippocampal system. Journal of Cognitive Neuroscience, $10,377-394$.

MacKay, D. G., Stewart, R., \& Burke, D. M. (1998b). H.M.'s language production deficits: Implications for relations between memory, semantic binding, and the hippocampal system. Journal of Memory and Language, 38, 28-69.

MacKay, D. G., James, L. E., \& Hadley, C. B. (2007). Amnesic H.M.'s performance on the language competence test: Parallel deficits in memory and sentence production. Journal of Clinical and Experimental Neuropsychology, 30, 280-300.

MacKay, D. G., James, L. E., Taylor, J. K., \& Marian, D. E. (2007). Amnesic H.M. exhibits parallel deficits and sparing of language and memory: Systems versus binding theory accounts. Language and Cognitive Processes, 22, 377-452.

Maclean, C. J., Gaffan, D., Baker, H. F., \& Ridley, R. M. (2001). Visual discrimination learning impairments produced by combined transections of the anterior temporal stem, amygdala and fornix in marmoset monkeys. Brain Research, 888, 34-50.

Maguire, E. A., Nannery, R., \& Spiers, H. J. (2006). Navigation around London by a taxi driver with bilateral hippocampal lesions. Brain, 129, 2894-2907.

Majerus, S., Norris, D., \& Patterson, K. (2007). What does a patient with semantic dementia remember in verbal short-term memory? Order and sound but not words. Cognitive Neuropsychology, 24, 131-151.

Manns, J. R., \& Squire, L. R. (2001). Perceptual learning, awareness, and the hippocampus. Hippocampus, 11, 776-782.

Manns, J. R., Hopkins, R. O., Reed, J. M., Kitchener, E. G., \& Squire, L. R. (2003). Recognition memory and the human hippocampus. Neuron, 37, 171-180.

Manns, J. R., Howard, M. W., \& Eichenbaum, H. (2007). Gradual changes in hippocampal activity support remembering the order of events, 56, 530-540.

Mayes, A. R., Holdstock, J. S., Isaac, C. L., Hunkin, N. M., \& Roberts, N. (2002). Relative sparing of item recognition memory in a patient with adult-onset damage limited to the hippocampus. Hippocampus, 12, 325-340.

Mayes, A. R., Montaldi, D., \& Migo, E. (2007). Associative memory and the medial temporal lobes. Trends in Cognitive Sciences, 11, 126-135.

McMackin, D., Cockburn, J., Anslow, P., \& Gaffan, D. (1995). Correlation of fornix damage with memory impairment in six cases of colloid cyst removal. Acta Neurochirurgica, 135, 12-18.

Meunier, M., Bachevalier, J., Mishkin, M., \& Murray, E. A. (1993). Effects on visual recognition of combined and separate ablations of the entorhinal and perirhinal cortex in rhesus monkeys. Journal of Neuroscience, 13, 5418-5432. 
Mishkin, M., Suzuki, W. A., Gadian, D. G., \& Vargha-Khadem, F. (1997). Hierarchical organization of cognitive memory. Philosophical Transactions of the Royal Society London. B Biological Sciences, 352, 1461-1467.

Mitchell, K. J., Johnson, M. K., Raye, C. L., \& D’Esposito, M. (2000). fMRI evidence of age-related hippocampal dysfunction in feature binding in working memory. Cognitive Brain Research, 10, 197-206.

Montaldi, D., Spencer, T. J., Roberts, N., \& Mayes, A. R. (2006). The neural system that mediates familiarity memory. Hippocampus, 16, 504-520.

Moscovitch, M., Nadel, L., Winocur, G., Gilboa, A., \& Rosenbaum, S. E. (2006). The cognitive neuroscience of remote episodic, semantic and spatial memory. Current Opinions in Neurobiology, 16, 179-190.

Moses, S. N., \& Ryan, J. D. (2006). A comparison and evaluation of the predictions of relational and conjunctive accounts of hippocampal function. Hippocampus, 16 , 43-65.

Moss, H. E., Rodd, J. M., Stamatakis, E. A., Bright, P., \& Tyler, L. K. (2005). Anteromedial temporal cortex supports fine-grained differentiation among objects. Cerebral Cortex, 15, 616-627.

Mumby, D. G., Mana, M. J., Pinel, J. P., David, E., \& Banks, K. (1995). Pyrithlamineinduced thiamine deficiency impairs object recognition in rats. Behavioural Neuroscience, 109, 1209-1214.

Mundy, M. E., Honey, R. C., Downing, P. E., Wise, R. G., Graham, K. S., \& Dwyer, D. M. (2009). Material-independent and material-specific activation in functional MRI after perceptual learning. Neuroreport, 20, 1397-1401.

Mundy, M.E., Downing, P.E., Dwyer, D.M., Honey, R.C. \& Graham, K.S. (submitted for publication). Material matters: Domain-specific discrimination learning for visually similar faces and scenes in perirhinal cortex and posterior hippocampus. Nature Neuroscience.

Murray, E. A. (1996). What have ablation studies told us about the neural substrates of stimulus memory? Seminars in the Neurosciences, 8, 13-22.

Murray, E. A., \& Bussey, T. J. (1999). Perceptual-mnemonic functions of the perirhinal cortex. Trends in Cognitive Sciences, 3, 142-151.

Murray, E. A., \& Mishkin, M. (1986). Visual recognition in monkeys following rhinal cortical ablations combined with either amygdalectomy or hippocampectomy. Journal of Neuroscience, 6, 1991-2003.

Murray, E. A., Saksida, L. M., \& Bussey, T. J. (2007). Visual perception and memory: A new view of medial temporal lobe function in primates and rodents. Annual Review of Neuroscience, 30, 99-122.

Nestor, P. J., Fryer, T. D., Ikeda, M., \& Hodges, J. R. (2003). Retrosplenial cortex (BA 29/30) hypometabolism in mild cognitive impairment (prodromal Alzheimer's disease). European Journal of Neuroscience, 18, 1-5.

Nestor, P. J., Fryer, T. D., Smielewski, P., \& Hodges, J. R. (2003). Limbic hypometabolism in Alzheimer's disease and mild cognitive impairment. Annuals of Neurology, 54, 343-351.

Nestor, P. J., Fryer, T. D., \& Hodges, J. R. (2006). Declarative memory impairments in Alzheimer's disease and semantic dementia. Neuroimage, 30, 1010-1020.

O'Neil, E. B., Cate, A. D., \& Kohler, S. (2009). Perirhinal cortex contributes to accuracy in recognition memory and perceptual discriminations. Journal of Neuroscience, 29, 8329-8334.

O'Keefe, J. (1976). Place units in the hippocampus of the freely moving rat. Experimental Neurology, 51, 78-109.

O'Keefe, J. (1991). An allocentric spatial model for the hippocampal cognitive map. Hippocampus, 1, 230-235.

O'Keefe, J., \& Burgess, N. (1996). Geometric determinants of the place fields of hippocampal neurons. Nature, 381, 425-428.

O’Keefe, J., Burgess, N., Donnett, J. G., Jeffery, K. J., \& Maguire, E. A. (1998). Place cells, navigational accuracy, and the human hippocampus. Philosophical Transactions of the Royal Society London. B Biological Sciences, 353, 1333-1340.

Olson, I. R., Moore, K. S., Stark, M., \& Chatterjee, A. (2006). Visual working memory is impaired when the medial temporal lobe is damaged. Journal of Cognitive Neuroscience, 18, 1087-1097.

Olson, I. R., Page, K., Moore, K. S., Chatterjee, A., \& Verfaellie, M. (2006). Working memory for conjunctions relies on the medial temporal lobe. Journal of Neuroscience, 26, 4596-4601.

Owen, A. M., Sahakian, B. J., Semple, J., Polkey, C. E., \& Robbins, T. W. (1995). Visuo-spatial short-term recognition memory and learning after temporal lobe excisions, frontal lobe excisions or amygdalo-hippocampectomy in man. Neuropsychologia, 33, 1-24.

Parker, A., \& Gaffan, D. (1998). Interaction of frontal and perirhinal cortices in visual object recognition memory in monkeys. European Journal of Neuroscience, 10, 3044-3057.

Patterson, K. (2007). The reign of typicality in semantic memory. Philosophical Transactions of the Royal Society London. Biological Sciences, 362, 813-821.

Patterson, K., Lambon Ralph, M. A., Jefferies, E., Woollams, A., Jones, R., Hodges, J. R., et al. (2006). "Presemantic" cognition in semantic dementia: Six deficits in search of an explanation. Journal of Cognitive Neuroscience, 18, 169-183.

Patterson, K., Nestor, P. J., \& Rogers, T. T. (2007). Where do you know what you know? The representation of semantic knowledge in the human brain. Nature Reviews Neuroscience, 8, 976-987.

Pihlajamaki, M., Tanila, H., Kononen, M., Hanninen, T., Hamalainen, A., Soininen, H., et al. (2004). Visual presentation of novel objects and new spatial arrangements of objects differentially activates the medial temporal lobe subareas in humans. European Journal of Neuroscience, 19, 1939-1949.

Preston, A. R., Bornstein, A. M., Hutchinson, J. B., Gaare, M. E., Glover, G. H., \& Wagner, A. D. (2009). High-resolution fMRI of content-sensitive subsequent memory responses in human medial temporal lobe. Journal of Cognitive Neuroscience [EPub Jan 13].
Prince, S. E., Dennis, N. A., \& Cabeza, R. (2009). Encoding and retrieving faces and places: Distinguishing process- and stimulus-specific differences in brain activity. Neuropsychologia, 47, 2282-2289.

Ranganath, C., \& Blumenfeld, R. S. (2005). Doubts about double dissociations between short- and long-term memory. Trends in Cognitive Sciences, 9 $374-380$.

Ranganath, C., \& D'Esposito, M. (2001). Medial temporal lobe activity associated with active maintenance of novel information. Neuron, 31, 865-873.

Ranganath, C., Yonelinas, A. P., Cohen, M. X., Dy, C. J., Tom, S. M., \& D’Esposito, M. (2004). Dissociable correlates of recollection and familiarity within the medial temporal lobes. Neuropsychologia, 42, 2-13.

Reed, J. M., \& Squire, L. R. (1997). Impaired recognition memory in patients with lesions limited to the hippocampal formation. Behavioural Neuroscience, 111 667-675.

Rogers, T. T., Patterson, K., \& Graham, K. S. (2007). Colour knowledge in semantic dementia: It's not all black and white. Neuropsychologia, 14, 3285-3298.

Rosenbaum, R. S., Priselac, S., Kohler, S., Black, S. E., Gao, F., Nadel, L., et al. (2000) Remote spatial memory in an amnesic person with extensive bilateral hippocampal lesions. Nature Neuroscience, 3, 1044-1048.

Rosenbaum, R. S., Gao, F., Richards, B., Black, S. E., \& Moscovitch, M. (2005). “Where to?" Remote memory for spatial relations and landmark identity in former tax drivers with Alzheimer's disease and encephalitis. Journal of Cognitive Neuroscience, $17,446-462$.

Rosenbaum, R. S., Moscovitch, M., Foster, J. K., Schnyer, D. M., Gao, F., Kovacevic, N., et al. (2008). Patterns of autobiographical memory loss in medial-temporal lobe amnesic patients. Journal of Cognitive Neuroscience, 20, 1490-1506.

Ryan, J. D., \& Cohen, N. J. (2004). The nature of change detection and online representations of scenes. Journal of Experimental Psychology Human Perception and Performance, 30, 988-1015.

Ryan, J. D., Althoff, R. R., Whitlow, S., \& Cohen, N. J. (2000). Amnesia is a deficit in relational memory. Psychological Science, 11, 454-461.

Saksida, L. M., Bussey, T. J., Buckmaster, C. A., \& Murray, E. A. (2006). No effect of hippocampal lesions on perirhinal cortex-dependent feature-ambiguous visual discriminations. Hippocampus, 16, 421-430.

Sauvage, M. M., Fortin, N. J., Owens, C. B., Yonelinas, A. P., \& Eichenbaum, H. (2008) Recognition memory: Opposite effects of hippocampal damage on recollection and familiarity. Nature Neuroscience, 11, 16-18.

Schacter, D. L., Chiu, C. Y., \& Ochsner, K. N. (1993). Implicit memory: A selective review. Annual Reviews Neuroscience, 16, 159-182.

Scoville, W. B. (1954). The limbic lobe in man. Journal of Neurosurgery, 11, 64-66.

Scoville, W. B., \& Milner, B. (1957). Loss of recent memory after bilateral hippocampal lesions. Journal of Neurology, Neurosurgery and Psychiatry, 20, 11-21.

Shrager, Y., Gold, J. J., Hopkins, R. O., \& Squire, L. R. (2006). Intact visual perception in memory-impaired patients with medial temporal lobe lesions. Journal of Neuroscience, 26, 2235-2240.

Shrager, Y., Levy, D. A., Hopkins, R. O., \& Squire, L. R. (2008). Working memory and the organization of brain systems. Journal of Neuroscience, 28 4818-4822.

Shrager, Y., Kirwan, C. B., \& Squire, L. R. (2008). Activity in both hippocampus and perirhinal cortex predicts the memory strength of subsequently remembered information. Neuron, 28, 547-553.

Sidman, M., Stoddard, L. T., \& Mohr, J. P. (1968). Some additional quantitative observations of immediate memory in a patient with bilateral hippocampal lesions. Neuropsychologia, 6, 245-254.

Simons, J. S., Verfaellie, M., Galton, C. J., Miller, B. L., Hodges, J. R., \& Graham, K. S (2002). Recollection-based memory in frontotemporal dementia: Implications for theories of long-term memory. Brain, 125, 2523-2536.

Spiers, H. J., \& Maguire, E. A. (2007). The neuroscience of remote spatial memory: A tale of two cities. Neuroscience, 149, 7-27.

Spiers, H. J., Burgess, N., Hartley, T., Vargha-Khadem, F., \& O’Keefe, J. (2001). Bilateral hippocampal pathology impairs topographical and episodic memory but not visual pattern matching. Hippocampus, 11, 715-725.

Squire, L. R. (1982). The neuropsychology of human memory. Annual Reviews in Neuroscience, 5, 241-273.

Squire, L. R. (2009). The legacy of patient H.M. for neuroscience. Neuron, 61 6-9.

Squire, L. R., \& Alvarez, P. (1995). Retrograde amnesia and memory consolidation: A neurobiological perspective. Current Opinion in Neurobiology, 5, 169-177.

Squire, L. R., \& Zola-Morgan, S. (1991). The medial temporal lobe memory system. Science, 253, 1380-1386.

Squire, L. R., Stark, C. E., \& Clark, R. E. (2004). The medial temporal lobe. Annual Reviews in Neuroscience, 27, 279-306.

Squire, L. R., Shrager, Y., \& Levy, D. A. (2006). Lack of evidence for a role of medial temporal lobe structures in visual perception. Learning and Memory, 13 106-107.

Squire, L. R., Wixted, J. T., \& Clark, R. E. (2007). Recognition memory and the medial temporal lobe: A new perspective. Nature Reviews Neuroscience, 8 , 872-883.

Staresina, B. P., \& Davachi, L. (2008). Selective and shared contributions of the hippocampus and perirhinal cortex to episodic item and associative encoding. Journal of Cognitive Neuroscience, 20, 1478-1489.

Stark, C. E., \& Okado, Y. (2003). Making memories without trying: Medial temporal lobe activity associated with incidental memory formation during recognition. Journal of Neuroscience, 23, 6748-6753.

Stark, C. E., \& Squire, L. R. (2000). Intact visual perceptual discrimination in humans in the absence of perirhinal cortex. Learning and Memory, 7, 273-278. 
Stark, C. E., Bayley, P. J., \& Squire, L. R. (2002). Recognition memory for single items and for associations is similarly impaired following damage to the hippocampal region. Learning and Memory, 9, 238-242.

Summerfield, J. J., Lepsien, J., Gitelman, D. R., Mesulam, M. M., \& Nobre, A. C. (2006). Orienting attention based on long-term memory experience. Neuron, $49,905-916$.

Suzuki, W. A. (2009). Perception and the medial temporal lobe: Evaluating the current evidence. Neuron, 61, 657-666.

Suzuki, W. A., \& Baxter, M. G. (2009). Memory, perception and the medial temporal lobe: A synthesis of opinions. Neuron, 61, 678-679.

Suzuki, W. A., Zola-Morgan, S., Squire, L. R., \& Amaral, D. G. (1993). Lesions of the perirhinal and parahippocampal cortices in the monkey produce long-lasting memory impairment in the visual and tactual modalities. Journal of Neuroscience, $13,2430-2451$.

Tanaka, J. W., \& Farah, M. J. (1991). Parts and wholes in face recognition. Quarterly Journal of Experimental Psychology, 46, 225-245.

Taylor, K. I., Moss, H. E., Stamatakis, E. A., \& Tyler, L. K. (2006). Binding crossmodal object features in perirhinal cortex. Proceedings of the National Academy of Sciences, USA, 103, 8239-8244.

Taylor, K. J., Henson, R. N. A., \& Graham, K. S. (2007). Recognition memory for faces and scenes in amnesia: Dissociable roles of medial temporal lobe structures. Neuropsychologia, 45, 2135-2146.

Teng, E., \& Squire, L. R. (1999). Memory for places learned long ago is intact after hippocampal damage. Nature, 400, 675-677.

Torralba, A., Oliva, A., Castelhano, M. S., \& Henderson, J. M. (2006). Contextual guidance of eye movements and attention in real-world scenes: The role of global features in object search. Psychological Review, 113, 766-786.

Tsivilis, D., Vann, S. D., Denby, C., Roberts, N., Mayes, A. R., Montaldi, D., et al. (2008). A disproportionate role for the fornix and mammillary bodies in recall versus recognition memory. Nature Neuroscience, 11, 834-842.

Turriziani, P., Fadda, L., Caltagirone, C., \& Carlesimo, G. A. (2004). Recognition memory for single items and for associations in amnesic patients. Neuropsychologia, $42,426-433$.

Tyler, L. K., Stamatakis, E. A., Bright, P., Acres, K., Abdallah, S., Rodd, J. M., et al. (2004). Processing objects at different levels of specificity. Journal of Cognitive Neuroscience, 16, 351-362.
Vargha-Khadem, F., Gadian, D. G., Watkins, K. E., Connelly, A., Van Paesschen, W., \& Mishkin, M. (1997). Differential effects of early hippocampal pathology on episodic and semantic memory. Science, 18, 376-380.

Viskontas, I. V., Carr, V. A., Engel, S. A., \& Knowlton, B. J. (2009). The neural correlates of recollection: Hippocampal activation declines as episodic memory fades. Hippocampus, 19, 265-272.

Wais, P. E., Wixted, J. T., Hopkins, R. O., \& Squire, L. R. (2006). The hippocampus supports both the recollection and the familiarity components of recognition memory. Neuron, 49, 459-466.

Winters, B. D., \& Bussey, T. J. (2005). Removal of cholinergic input to perirhinal cortex disrupts object recognition but not spatial working memory in the rat. European Journal of Neuroscience, 21, 2263-2270.

Winters, B. D., Saksida, L. M., \& Bussey, T. J. (2008). Object recognition memory: Neurobiological mechanisms of encoding, consolidation and retrieval. Neuroscience Biobehavioural Reviews, 32, 1055-1070.

Wise, S. P., Murray, E. A., \& Gerfen, C. R. (1996). The frontal cortex-basal ganglia system in primates. Critical Reviews in Neurobiology, 10, 317-356.

Wixted, J. T. (2007). Dual-process theory and signal-detection theory of recognition memory. Psychological Review, 114, 152-176.

Yonelinas, A. P., Kroll, N. E., Dobbins, I. G., \& Soltani, M. (1999). Recognition memory for faces: When familiarity supports recognition judgments. Psychonomics Bulletin Reviews, 6, 654-661.

Yonelinas, A. P., Kroll, N. E., Quamme, J. R., Lazzara, M. M., Sauve, M. J., Widaman, K. F., et al. (2002). Effects of extensive temporal lobe damage or mild hypoxia on recollection and familiarity. Nature Neuroscience, 5, 1236-1241.

Young, A. W., Hellawell, D., \& Hay, D. C. (1987). Configurational information in face perception. Perception, 16, 747-759.

Zhu, X. O., Brown, M. W., McCabe, B. J., \& Aggleton, J. P. (1995). Effects of the novelty or familiarity of visual stimuli on the expression of the immediate early gene c-fos in rat brain. Neuroscience, 69, 821-829.

Zhu, X. O., McCabe, B. J., Aggleton, J. P., \& Brown, M. W. (1996). Mapping recognition memory through the differential expression of the immediate early gene c-fos induced by novel or familiar visual stimulation. NeuroReport, 7, 1871-1875.

Zhu, X. O., McCabe, B. J., Aggleton, J. P., \& Brown, M. W. (1997). Differential activation of the hippocampus and perirhinal cortex by novel visual stimuli and a novel environment. Neuroscience Letters, 229, 141-143. 